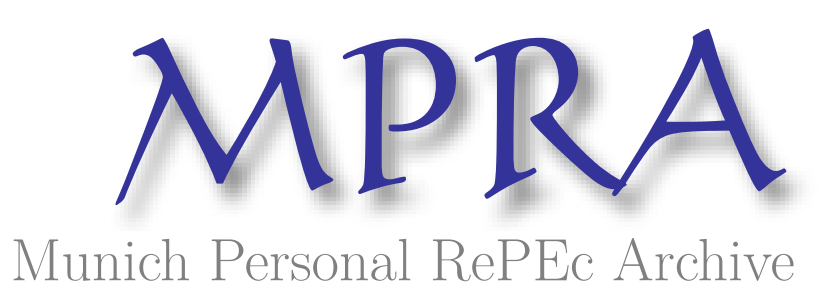

\title{
Closed-Form Approximations for Spread Option Prices and Greeks
}

Li, Minqiang

2008

Online at https://mpra.ub.uni-muenchen.de/6994/

MPRA Paper No. 6994, posted 06 Feb 2008 05:53 UTC 


\title{
Closed-form Approximations for Spread Option Prices and Greeks
}

\author{
Shi-Jie Deng*, Minqiang Lił Jieyun Zhou
}

January 21, 2008

\begin{abstract}
We develop a new closed-form approximation method for pricing spread options. Numerical analysis shows that our method is more accurate than existing analytical approximations. Our method is also extremely fast, with computing time more than two orders of magnitude shorter than one-dimensional numerical integration. We also develop closed-form approximations for the greeks of spread options. In addition, we analyze the price sensitivities of spread options and provide lower and upper bounds for digital spread options. Our method enables the accurate pricing of a bulk volume of spread options with different specifications in real time, which offers traders a potential edge in financial markets. The closed-form approximations of greeks serve as valuable tools in financial applications such as dynamic hedging and Value-at-Risk calculations.
\end{abstract}

*755 Ferst Drive NW, School of Industrial and Systems Engineering, Georgia Institute of Technology, Atlanta, GA, 30332. E-mail: deng@gatech.edu.

${ }^{\dagger}$ Corresponding author. 800 West Peachtree Street, College of Management, Georgia Institute of Technology, Atlanta, GA, 30332. E-mail minqiang.li@mgt.gatech.edu. Phone: 404-894-4926. Fax: 404-894-6030.

${ }^{\ddagger} 755$ Ferst Drive NW, School of Industrial and Systems Engineering, Georgia Institute of Technology, Atlanta, GA, 30332. E-mail: jzhou@isye.gatech.edu. 


\section{INTRODUCTION}

Spread options allow investors to simultaneously take positions in two are more assets and profit from their price difference over some spread. Spread options are prevalent in equity, fixed income, foreign exchange and commodity markets. For instance, in the fixed income markets, various instruments are traded on exchanging securities with different maturities (such as Treasury Notes and Bonds), with different quality levels (such as the Treasury Bills and Eurodollars), and with different issuers (such as French and German bonds, or Municipal bonds and Treasury Bonds). In the agricultural markets, the CBOT trades the so-called crush spread which exchanges raw soybeans with a combination of soybean oil and soybean meal. In the energy markets, crack spread options, which either exchange crude oil and unleaded gasoline or exchange crude oil and heating oil, are traded on the NYMEX. Electricity spark spread options are also traded over the counter for exchanging a specific fuel for electricity. Many studies have focused on spread options in these markets. For example, Arak et al. (1987), Jones (1991), and Easterwood and Senchack (1986) study spread options in the fixed income markets. Johnson et al. (1991) study spread options in the agricultural markets. Girma and Paulson (1998, 1999) and Deng et al. (2001) study spread options in the energy markets.

We focus on spread options written on two assets in this paper. Spread options written on more than two assets is dealt with in a separate paper. The risk-neutral valuation of the two-asset spread option price involves a two-dimensional integration. We introduce a key concept called the exercise boundary. It is defined as the minimal log price of asset one, as a function of the log price of asset two, for the option to expire in the money. For convenience, we also standardize these two log prices. Under setups in which the asset returns are jointly normally distributed, closedform formula (Margrabe 1978) exists for pricing exchange options, which are spread options with zero spreads. The critical reason why such a closed-form formula can be obtained for exchange options is that the exercise boundary of a spread option is linear when the spread is zero, which

allows the double integration to be evaluated in closed form. However, for general spread options where the spread is not zero, the exercise boundary becomes nonlinear, which prevents people from obtaining a closed-form formula. Thus, it is a challenge to compute spread option prices efficiently and accurately as no exact closed-form formula exists for spread options with general nonzero spread.

Existing methods for pricing spread options can be roughly divided into two groups: numerical methods and analytical approximations. Numerical methods include numerical integration, Monte Carlo simulation, and fast Fourier transform. Analytical methods generally seek to obtain closed-form formula to approximate the spread option price. Various analytical methods have 
been proposed. In the Bachelier approximation (Wilcox 1990, Shimko 1994, Poitras 1998), one approximates the price difference of the two assets directly as a normal random variable and then uses the Bachelier formula for plain-vanilla options to approximate the spread option price. Unfortunately, the Bachelier approximation is found to be rather crude. Some attempts (Mbanefo (1997)) have been made to improve the accuracy of the Bachelier approximation, usually by including high-order moments of the price difference or using a Gram-Charlier density function pioneered in finance by Jarrow and Rudd (1982). Kirk (1995) uses the Margrabe formula to price spread options by combining the second asset and the fixed spread into a single asset which is then treated as lognormally distributed. His method is equivalent to a linearization of the nonlinear exercise boundary. This method is found to be relatively accurate and thus currently relatively popular among practitioners. Carmona and Durrleman (2003a, 2003b) design a new method to approximate the spread option price by giving the lower and upper price bounds. The CarmonaDurrleman method is generally more accurate than other analytical methods. However, a critical shortcoming is that in this method one needs to solve a nonlinear system of equations which is computationally costly and not completely trivial. Thus, unlike other analytical methods, the Carmona-Durrleman method does not give a closed-form formula for the spread option price.

However, there are weaknesses in the existing methods of both approaches. In general, while numerical methods are often accurate, their computing times are usually much longer than desirable. On the other hand, analytical approximation methods are generally faster than numerical methods but often lack accuracy and robustness. Thus, it is desirable to have a method that combines the strengths of existing methods while avoiding their weaknesses, namely, a method that is both accurate and fast.

The purpose of this paper is to derive closed-form approximations for the spread option price and greeks which are more accurate and faster than existing methods. We make several important contributions. First, we propose a new closed-form approximation for pricing spread options based on a quadratic approximation of the exercise boundary. Our approximation is extremely accurate, often resulting in relative pricing errors smaller than $10^{-4}$. Second, our approach differs from existing analytical approximations in that we approximate each term in the spread option price separately. Approximating individual terms allows us to compute digital-type spread options very accurately. More importantly, it also leads to extremely accurate approximations for the greeks, which are of significant importance in practical applications such as dynamic hedging and Value-at-Risk calculations. Third, we develop lower and upper bounds for digital spread options. Finally, we provide an analytical study on the price sensitivities of the spread options. In particular, we characterize the signs of the vegas when the correlation coefficient between the 
two underlyings is negative or positive and large.

Our closed-form approximation formula for spread option prices can offer insights on the designing and analyzing of real options embedded in financial and real contracts. Spread options with zero spread, a.k.a. exchange options, have been employed extensively by researchers to model real options, partly because of the availability of the Margrabe formula. For example, McDonald and Siegel (1985) use the Margrabe formula to study the investment and valuation of firms when there is an option to shut down. Shevlin (1991) investigates the valuation of $R \& D$ firms with $R \& D$ limited partnerships. Albizzati and Geman (1994) value the surrender option in life insurance polices by extending the Margrabe formula to a Heath-Jarrow-Morton stochastic interest rate framework. Grinblatt and Titman (1989), and Johnson and Tian (2000) apply the Margrabe formula to study the design and effectiveness of performance-based contracts and executive stock options. However, in many of these applications, it is more natural to assume that we have a spread option instead of an exchange option. The spread $K$ may correspond to the cost or salvage value of shutting down a firm, the cost or salvage value of terminating an $R \& D$ partnership, the monetary penalty of surrendering the life insurance policy prematurely, a minimal level $(K>0)$ of performance difference that a manager has to achieve over a benchmark, or a cushion $(K<0)$ to insure the manager that he will not be unfairly penalized because of pure bad luck. The extra degree of freedom arising from a nonzero $K$ could be extremely important in designing and analyzing these real options.

The paper is organized as follows. Section II discusses the general framework we use; that is, asset returns are jointly-normally distributed. We reduce the spread option pricing problem to a one-dimensional problem in Proposition 1, show that it reduces to the Margrabe formula when the exercise boundary is linear in Proposition 2, and discuss the properties of the exercise boundary for general spread options in Proposition 3. Section III develops lower and upper bounds for the digital spread option in Propositions 4 and 5 based on a tangent line approximation and a chord approximation of the exercise boundary, respectively. Section IV develops a closedform approximation for spread options in Proposition 6 based on a quadratic approximation of the exercise boundary. Proposition 6 is the central result of this paper. We also analyze the price sensitivities of spread options in our general framework in Proposition 7 and the special geometric Brownian motions case in Proposition 8. We then give closed-form approximations in Proposition 9 for the spread option greeks. Section V compares our method with existing analytical approximations and numerical integration in terms of speed and accuracy and shows that our method is both very accurate and fast. Section VI concludes and discusses possible extensions. Proofs are in the Appendix. 


\section{THE MODEL SETUP}

The main purpose of this paper is to derive an efficient and accurate method for computing spread option prices. Under the general assumption of jointly-normal returns, closed-form formula exists for exchange options, that is, spread options with the spread $K$ being zero. However, for general spread options with nonzero $K$, exact closed-form formula is not available. In this paper, we develop an extremely accurate analytical approximation for spread option prices with general values of $K$. The relative errors of our approximation are usually smaller than $10^{-4}$, well within the observed bid-ask spreads of these options.

We first describe the setup we will use for pricing spread options, that is, the returns of the two assets are jointly normally distributed. Specifically, consider two assets whose prices at time $t$ are denoted by $S_{1}(t)$ and $S_{2}(t)$. We are interested in options whose final payoffs are nonnegative only when $S_{1}(T)-S_{2}(T)-K \geq 0$ at some future time $T$, where the spread $K$ is a constant. We focus on cash-or-nothing digital spread options with time- $T$ payoff given by $1_{S_{1}(T) \geq S_{2}(T)+K}$, and spread options with time- $T$ payoff $\left[S_{1}(T)-S_{2}(T)-K\right]^{+}$, where we use $f^{+}$to denote the positive part of the function $f$. By the martingale pricing approach, the prices of a digital spread option $\Pi^{\mathrm{D}}$ and a spread option $\Pi$ are given by

$$
\Pi^{\mathrm{D}}=e^{-r T} \mathbb{E}^{\mathbb{Q}}\left[1_{\left\{S_{1}(T) \geq S_{2}(T)+K\right\}}\right], \quad \Pi=e^{-r T} \mathbb{E}^{\mathbb{Q}}\left[S_{1}(T)-S_{2}(T)-K\right]^{+},
$$

where $\mathbb{Q}$ is the risk-neutral measure under which discounted security prices are martingales.

To compute these option prices, distributional assumptions on $S_{1}(T)$ and $S_{2}(T)$ need to be made. We assume that $\log S_{1}(T)$ and $\log S_{2}(T)$ are jointly normally distributed. Specifically, let the initial prices of the two assets be $S_{1}(0)=S_{1}, S_{2}(0)=S_{2}$, and

$$
\mathbb{E}^{\mathbb{Q}}\left[\log S_{i}(T)\right]=\mu_{i}, \quad \operatorname{var}^{\mathbb{Q}}\left[\log S_{i}(T)\right]=\nu_{i}^{2}, \quad(i=1,2)
$$

where the means $\mu_{i}$ 's and the variances $\nu_{i}$ 's are all deterministic quantities. Next, we define

$$
X=\frac{\log S_{1}(T)-\mu_{1}}{\nu_{1}}, \quad Y=\frac{\log S_{2}(T)-\mu_{2}}{\nu_{2}} .
$$

Notice that $X$ and $Y$ are the standardized log prices of asset one and two, respectively. In our setup, we will assume that $X$ and $Y$ are jointly normal with correlation coefficient $\rho$ and with standard normal marginal densities.

This general setup incorporates two important cases, namely, the geometric Brownian motions (GBMs) case and the mean-reverting log-Ornstein-Uhlenbeck (log-OU) case. Specifically, let $W_{1}(t)$ and $W_{2}(t)$ be two Brownian motions with correlation $\varrho$. In the GBMs case, we have

$$
\mathrm{d} S_{i}(t)=\left(r-q_{i}\right) S_{i}(t) \mathrm{d} t+\sigma_{i} S_{i}(t) \mathrm{d} W_{i}(t),
$$


where $r$ is the risk-free interest rate, $\sigma_{i}$ 's are the volatilities, and $q_{i}$ 's are the dividend rates. A simple application of Ito's lemma tells us that $S_{1}(T)$ and $S_{2}(T)$ are jointly normally distributed, with the $\mu_{i}$ 's and $\nu_{i}$ 's in equation (2) given by

$$
\mu_{i}=\log S_{i}+\left(r-q_{i}-\sigma_{i}^{2} / 2\right) T, \quad \nu_{i}=\sigma_{i} \sqrt{T}, \quad \rho=\varrho,
$$

The GBMs case can be easily generalized to incorporate seasonality in parameters by allowing $\sigma_{i}$ 's, $q_{i}$ 's and $\rho$ be to deterministic functions of the calendar time $t$. This is useful since for some spread options, their underlying assets exhibit strong seasonality in price volatilities and in their return correlations. Our general framework incorporates this generalized GBMs case.

In the log-OU case, we have

$$
\mathrm{d} S_{i}(t)=-\lambda_{i}\left(\log S_{i}(t)-\eta_{i}\right) S_{i}(t) \mathrm{d} t+\sigma_{i} S_{i}(t) \mathrm{d} W_{i}(t),
$$

where $\lambda_{i}$ 's are the mean-reverting strengths and $\eta_{i}$ 's are parameters controlling the long-run means. With some algebra, it can be shown that $S_{1}(T)$ and $S_{2}(T)$ are jointly normally distributed, with the $\mu_{i}$ 's and $\nu_{i}$ 's in equation (2) given by

$$
\begin{gathered}
\mu_{i}=\eta_{i}-\frac{\sigma_{i}^{2}}{2 \lambda_{i}}+e^{-\lambda_{i} T}\left(\log S_{i}-\eta_{i}+\frac{\sigma_{i}^{2}}{2 \lambda_{i}}\right), \quad \nu_{i}=\sigma_{i} \sqrt{\frac{1-e^{-2 \lambda_{i} T}}{2 \lambda_{i}}}, \\
\rho=2 \varrho \frac{\sqrt{\lambda_{1} \lambda_{2}}}{\lambda_{1}+\lambda_{2}} \frac{1-e^{-\left(\lambda_{1}+\lambda_{2}\right) T}}{\sqrt{1-e^{-2 \lambda_{1} T}} \sqrt{1-e^{-2 \lambda_{2} T}}}
\end{gathered}
$$

Before introducing our method, we present a thorough analysis of the exercise boundary of the spread option. The exercise boundary is defined to be the minimal standardized log price of asset one for the option to be in the money as a function of the standardized log price of asset two. A detailed study on the exercise boundary is important because s we will see later, the existence of a closed-form formula for exchange options but not for general spread options results exactly from the linearity of the exercise boundary when the spread is zero.

At time $T$, the options are in-the-money if $S_{1}(T)-S_{2}(T)-K \geq 0$. Let $K \geq 0$. By the definitions of $X$ and $Y$ in equation (3), this condition is the same as

$$
X \geq \frac{\log \left(e^{\nu_{2} Y+\mu_{2}}+K\right)-\mu_{1}}{\nu_{1}}
$$

Thus, conditioning on $Y=y$, the option is in-the-money if $X \geq \underline{x}(y)$, where the (conditional) exercise boundary $\underline{x}(y)$ is given by

$$
\underline{x}(y) \equiv \frac{\log \left(e^{\nu_{2} y+\mu_{2}}+K\right)-\mu_{1}}{\nu_{1}} .
$$


When $K<0$, the condition in equation (9) is not always well-defined because it is possible that $e^{\nu_{2} Y+\mu_{2}}+K<0$. However, by making use of the identities

$$
\begin{gathered}
1_{S_{1}(T) \geq S_{2}(T)+K}=1-1_{S_{2}(T) \geq S_{1}(T)-K} \\
{\left[S_{1}(T)-S_{2}(T)-K\right]^{+}=S_{1}(T)-S_{2}(T)-K+\left(S_{2}(T)-S_{1}(T)+K\right)^{+}}
\end{gathered}
$$

we can transform the problems of computing $\Pi^{\mathrm{D}}$ and $\Pi$ in the $K<0$ case to the $K>0$ case. For example, by equation (11), to compute the price of a digital spread option with final payoff $1_{S_{1}(T) \geq S_{2}(T)+K}$ where $K<0$, we can switch the roles of $S_{1}$ and $S_{2}$ and compute the price of the digital spread option with final payoff $1_{S_{2}(T) \geq S_{1}(T)+|K|}$. Similarly, equation (12) allows us to only consider spread options with $K \geq 0$. Consequently, throughout this paper we assume that $K \geq 0$, so that equation (9) is always well-defined.

The risk-neutral valuation in equation $(1)$ gives $\Pi^{\mathrm{D}}$ and $\Pi$ in terms of two two-dimensional integrations. However, in the following proposition, we utilize a method introduced in Pearson (1995) which reduces the two-dimensional integrations to one-dimensional integrations. Reducing the two-dimensional integration problem in equation (1) to a one-dimensional integration is useful not only for our approximation later, but also for numerical methods. For example, if one uses numerical integration, then by Proposition 1, we only need to evaluate one-dimensional integrals, which is considerably faster than evaluating two-dimensional integrals. Also, Proposition 1 is useful for Monte Carlo simulation. Carrying out Monte Carlo directly using equation (1) is not very efficient because many realizations of $\left(S_{1}(T)-S_{2}(T)-K\right)^{+}$will be zero, especially for out-ofthe-money spread options. Also, we need to simulate a bivariate distribution. With Proposition 1 , we can simulate a single random variable $y$ using a standard normal density and then compute the $\mathbf{I}_{i}$ 's by taking the sample averages of the three cumulative normal distribution functions. In this way, information in each realization of $y$ is utilized and thus Proposition 1 plays a similar role as importance sampling in variance reduction.

Proposition 1. Under the jointly-normal returns setup, the prices of the spread option and the digital spread option are given by

$$
\Pi=e^{\nu_{1}^{2} / 2+\mu_{1}-r T} \mathbf{I}_{1}-e^{\nu_{2}^{2} / 2+\mu_{2}-r T} \mathbf{I}_{2}-K e^{-r T} \mathbf{I}_{3}, \quad \text { and } \quad \Pi^{\mathrm{D}}=e^{-r T} \mathbf{I}_{3},
$$

where the integrals $\mathbf{I}_{i}$ 's are given by

$$
\begin{aligned}
& \mathbf{I}_{1}=\int_{-\infty}^{\infty} N\left(A\left(y+\rho \nu_{1}\right)+\sqrt{1-\rho^{2}} \nu_{1}\right) n(y) \mathrm{d} y, \\
& \mathbf{I}_{2}=\int_{-\infty}^{\infty} N\left(A\left(y+\nu_{2}\right)\right) n(y) \mathrm{d} y, \\
& \mathbf{I}_{3}=\int_{-\infty}^{\infty} N(A(y)) n(y) \mathrm{d} y,
\end{aligned}
$$


where $n(\cdot)$ and $N(\cdot)$ are the standard normal density function and the cumulative normal distribution function, respectively, and the function $A(\cdot)$ is given by

$$
A(y)=\frac{\rho y-\underline{x}(y)}{\sqrt{1-\rho^{2}}}
$$

with the exercise boundary $\underline{x}(y)$ given in equation (10).

Equation (13) in Proposition 1 gives a formula for the spread option price very similar to the Black-Scholes formula (Black and Scholes 1973). In particular, the price of the spread option $\Pi$ consists of three terms. The first term is the present value of the risk-neutral expected future benefit of receiving asset one. The second term is the present value of expected future cost of giving up asset two if the option expires in the money. The last term is the present value of the expected cost of giving up an additional monetary amount $K$.

The quantities $A(y)$ and $\mathbf{I}_{i}$ 's have intuitive meanings. We will call $A(y)$ the conditional moneyness of the spread option because $A(y)$ plays a similar role as $d_{2}$ in the Black-Scholes formula. This can be seen from the proof of Proposition 1 that the quantity $N(A(y))$ is the risk-neutral probability that the spread option expires in the money conditioning on that the standardized log price of asset two is $y$. Written out explicitly, we have

$$
N(A(y))=\operatorname{Prob}^{\mathbb{Q}}\left[S_{1}(T) \geq S_{2}(T)+K \mid Y \equiv \frac{\log S_{2}(T)-\mu_{2}}{\nu_{2}}=y\right] .
$$

In the Black-Scholes formula, $y$ is a constant, while in the case of spread options, $y$ is distributed as a standard normal random variable. Integrating over $y$ in equation (16) then gives the unconditional exercise probability $\mathbf{I}_{3}$. That is, $\mathbf{I}_{3}$ is the probability that the spread option will expire in the money under the risk-neutral distribution. The proof of Proposition 1 shows that $\mathbf{I}_{1}$ and $\mathbf{I}_{2}$ have similar meanings as $\mathbf{I}_{3}$. They are the probabilities that the spread option will expire in the money under the two measures in which asset one and asset two are taken to be the numeraire asset, respectively. This is similar to the case of the Black-Scholes formula, where the term $N\left(d_{1}\right)$ is the probability that the option will expire in the money under the probability measure in which the underlying stock is taken as the numeraire asset. For general change of numeraire technique, see Geman et al. (1995). The quantities $\mathbf{I}_{i}$ 's are also related to the greeks. As we will see later, in the GBMs case, $\mathbf{I}_{1}$ and $-\mathbf{I}_{2}$ are also the deltas of the spread option price with respect to the initial prices of asset one and asset two, respectively, while $\mathbf{I}_{3}$ is related to the price sensitivity of the spread option with respect to the spread $K$.

In Proposition 1, we have given the spread option price in terms of three one-dimensional integrals $\mathbf{I}_{1}, \mathbf{I}_{2}$ and $\mathbf{I}_{3}$. In the rest of the paper, we will develop closed-form approximations based 
on Proposition 1. We carry this out in a few steps. First, in Proposition 2 below, we will show that these integrals can be computed in closed form to yield the Margrabe formula if the exercise boundary $\underline{x}(y)$ (and hence the conditional moneyness function $A(y)$ ) is linear, which happens exactly when the spread $K$ is zero. Second, we study the monotonicity and convexity properties of the exercise boundary and the conditional moneyness function in detail in Proposition 3. Finally, in later sections, we approximate the exercise boundary by making use of these properties. These approximations in turn allow us to derive price bounds for digital options in Proposition 4 and 5, as well as approximate spread option prices in Proposition 6.

We first take a look at the special case when $K=0$. Proposition 2 derives the Margrabe formula (Margrabe 1978) for this special case using a new mathematical identity (equation 19), which will also be very useful for our approximation later on. If we interpret $n\left(y ; \mu, \sigma^{2}\right)$ in the proposition as the density of the log price of asset two and $a+b y$ as the conditional moneyness $A(y)$, then this identity says that the unconditional moneyness can be computed in closed form when the conditional moneyness $A(y)$ is a linear function of $y$. For spread options, from the expression for the exercise boundary $\underline{x}(y)$ in equation (10), we see that $\underline{x}(y)$ is linear in $y$ precisely when $K=0$. Notice also that $A(y)$ is linear in $y$ if and only if $\underline{x}(y)$ is linear in $y$. Thus, Proposition 2 offers a direct proof of the Margrabe formula which differs from the original partial differential equation approach in Margrabe (1978). Also, the Margrabe formula we give is more general than the original form as it applies to all models in which the returns are jointly normally distributed, and just the GBMs case. The proof of Proposition 2 is given in the Appendix.

Proposition 2. Let $a$ and $b$ be real numbers. Then we have

$$
\int_{-\infty}^{\infty} N(a+b y) n\left(y ; \mu, \sigma^{2}\right) \mathrm{d} y=N\left(\frac{a+b \mu}{\sqrt{1+b^{2} \sigma^{2}}}\right) .
$$

For exchange options (spread options with $K=0$ ), the conditional moneyness function $A(y)$ is linear in y. Thus, from equation (19), the price of an exchange option under the jointly-normal returns setup is given by the following Margrabe formula

$$
\Pi=e^{\nu_{1}^{2} / 2+\mu_{1}-r T} N\left(\frac{\mu_{1}-\mu_{2}+\left(\nu_{1}^{2}-\rho \nu_{1} \nu_{2}\right)}{\sqrt{\nu_{1}^{2}+\nu_{2}^{2}-2 \rho \nu_{1} \nu_{2}}}\right)-e^{\nu_{2}^{2} / 2+\mu_{2}-r T} N\left(\frac{\mu_{1}-\mu_{2}-\left(\nu_{2}^{2}-\rho \nu_{1} \nu_{2}\right)}{\sqrt{\nu_{1}^{2}+\nu_{2}^{2}-2 \rho \nu_{1} \nu_{2}}}\right) .
$$

For general spread options, $K \neq 0$. An immediate difficulty to apply Proposition 2 in this case is that the arguments for the cumulative normal distributions in the integrals $\mathbf{I}_{1}, \mathbf{I}_{2}$ and $\mathbf{I}_{3}$, namely, $A\left(y+\rho \nu_{1}\right)+\sqrt{1-\rho^{2}} \nu_{1}, A\left(y+\nu_{2}\right)$ and $A(y)$, are not linear functions of $y$. From the expression for $A(y)$, we see that this is precisely because the exercise boundary $\underline{x}(y)$ is not linear in $y$. However, a closer examination reveals that $\underline{x}(y)$ is quite close to linear locally. When $y$ is 
very negative, $\underline{x}(y)$ behaves like a constant function. When $y$ is very positive, $\underline{x}(y)$ behaves like a linear function. Exhibit 1 plots the function $\underline{x}(y)$ for different values of $K$. The parameters used are for the GBMs case with $S_{1}=90, S_{2}=100, r=5 \%, q_{1}=q_{2}=0, \sigma_{1}=0.4, \sigma_{2}=0.5$ and $T=0.25$. The idea of our approximation is to approximate the exercise boundary $\underline{x}(y)$ using a lower-order Taylor expansion, for example, a linear or quadratic function. This has the advantage that we approximate the $\mathbf{I}_{i}$ 's separately. Notice that $\underline{x}(y)$ is exactly linear when either $\sigma_{2}=0$ or $K=0$. Thus, as we shall see, our approximation is exact when $\sigma_{2}=0$ or $K=0$. This is appealing because when $\sigma_{2}=0$, our formula collapses to the Black-Scholes formula for an ordinary European call option, and when $K=0$, our formula collapses to the Margrabe formula for an exchange option.

\section{Insert Exhibit 1 Approximately Here}

Because our approximation tries to catch the deviation of the exercise boundary from linearity, a closer look at the regions of monotonicity and convexity of the exercise boundary $\underline{x}(y)$, the riskneutral conditional moneyness $A(y)$, and the conditional exercise probability $N(A(y))$ is crucial. In addition, by making use of the monotonicity and convexity properties, we are able to look at price bounds for digital spread options as we will do in the next section. The results of this monotonicity and convexity analysis are given in the following proposition. For readers who are less interested in the mathematical details, it suffices to understand the following three points from Proposition 3. First, the exercise boundary $\underline{x}(y)$ is convex in $y$ while the conditional moneyness function $A(y)$ is concave in $y$. Second, the behavior of $A(y)$ and $N(A(y))$ is influenced by the sign and size of the correlation coefficient $\rho$. Third, the behavior of $A(y)$ and $N(A(y))$ could be different for different regions of $y$. These properties will be used later on. For example, as we will see later, the sign and size of $\rho$ has influence on the signs of spread option vegas. The proof of Proposition 3 is given in the Appendix.

Proposition 3. Let $K \geq 0$ and $|\rho|<1$.

1. The exercise boundary $\underline{x}(y)$ is an increasing and convex function of $y$.

2. If $\rho \geq \nu_{2} / \nu_{1}$, the conditional moneyness $A(y)$ and risk-neutral conditional exercise probability $N(A(y))$ are both monotonically increasing in $y$. If $\rho \leq 0, A(y)$ and $N(A(y))$ are both monotonically decreasing in $y$. If $0<\rho<\nu_{2} / \nu_{1}$, both $A(y)$ and $N(A(y))$ have exactly one 
maximum at

$$
\widehat{y}=\frac{1}{\nu_{2}} \log \left(\frac{e^{-\mu_{2}} K \rho \nu_{1}}{\nu_{2}-\rho \nu_{1}}\right)
$$

3. The conditional moneyness $A(y)$ is a concave function of $y$.

4. If $e^{\mu_{1}+\rho \nu_{1} y}>e^{\mu_{2}+\nu_{2} y}+K$, then the conditional exercise probability $N(A(y))$ is locally concave at $y$. Furthermore, suppose the solution of $e^{\mu_{1}+\rho \nu_{1} y}=e^{\mu_{2}+\nu_{2} y}+K$ exists and denote it by $\widetilde{y}$. Then $N(A(y))$ is concave in the region $(\widetilde{y},+\infty)$ if $\nu_{2}<\rho \nu_{1}$, and concave in the region $(-\infty, \widetilde{y})$ if $\rho<0$.

Now that we have studied the monotonicity and convexity properties of the conditional moneyness $A(y)$ in Proposition 3, we are ready to develop our approximations. In the next section, we establish lower and upper bounds on the digital spread option with the help of Proposition 2 and 3. Readers who are interested in spread option prices can go directly to Section IV where we develop closed-form approximations for spread option prices.

\section{BOUNDS FOR DIGITAL SPREAD OPTION PRICES}

\section{A. The Upper Bound - Tangent Line Approximation}

In this section, we derive upper and lower bounds for digital spread options by approximating the exercise boundary. Pricing bounds for spread options can be obtained similarly. Pricing bounds are useful when no closed-form formula exists. Early studies in this area include Perrakis and Ryan (1984) and Lo (1987). More recently, Nielsen and Sandmann (2003), and Henderson, et al. (2007) derive pricing bounds for Asian options. Pricing bounds for American options are studied in Broadie and Detemple (1996), Chen and Yeh (2002), Chung and Chang (2007), among others. Carmona and Durrleman (2006) study pricing bounds for spread options.

By approximating the exercise boundary $\underline{x}(y)$ more or less favorably, we can establish price bounds for digital spread options. We look at the upper bound first. By Proposition 3, the conditional moneyness $A(y)$ is concave in $y$. Thus, if we draw any tangent line of $A(y)$, it will lie completely above the graph of $A(y)$. Pick an arbitrary point $y_{0}$. By the expression of $A(y)$ in equation (17), the tangent line of $A(y)$ at $y_{0}$ is given by $G\left(y_{0}\right)+H\left(y_{0}\right) y$, where

$$
\begin{aligned}
& G\left(y_{0}\right)=\frac{1}{\nu_{1} \sqrt{1-\rho^{2}}}\left(\mu_{1}-\log (R+K)+\frac{R}{R+K} \nu_{2} y_{0}\right), \\
& H\left(y_{0}\right)=\frac{1}{\sqrt{1-\rho^{2}}}\left(\rho-\frac{R \nu_{2}}{(R+K) \nu_{1}}\right),
\end{aligned}
$$


with

$$
R=e^{\nu_{2} y_{0}+\mu_{2}} .
$$

For any tangent position $y_{0}$, the conditional moneyness approximation $G\left(y_{0}\right)+H\left(y_{0}\right) y$ is more favorable than the actual conditional moneyness $A(y)$. Proposition 1 and 2 now give us the following inequality:

$$
\mathbf{I}_{\mathbf{3}}=\int_{-\infty}^{\infty} N(A(y)) n(y) \mathrm{d} y \leq \int_{-\infty}^{\infty} N\left(G\left(y_{0}\right)+H\left(y_{0}\right) y\right) n(y) \mathrm{d} y=N\left(\frac{G\left(y_{0}\right)}{\sqrt{1+H^{2}\left(y_{0}\right)}}\right) .
$$

Since the above derivation is valid for any $y_{0}$, we can establish an upper bound for the price of a digital spread option:

Proposition 4. Let $K \geq 0$ and $|\rho|<1$. Then

$$
\Pi^{\mathrm{D}} \leq \inf _{y_{0} \in \mathbb{R}} e^{-r T} N\left(\frac{G\left(y_{0}\right)}{\sqrt{1+H^{2}\left(y_{0}\right)}}\right) .
$$

In the above proposition, each candidate in the infimum is the price of a digital option whose exercise boundary is more favorable than the one in our digital spread option. Different values of $y_{0}$ match the slopes of the actual and approximating exercise boundaries at different future $\log$ prices of asset two. For example, setting $y_{0}=0$ amounts to matching the slope of the exercise boundary exactly when the log price of asset two equals its mean. The two limiting cases $y_{0} \rightarrow \pm \infty$ correspond to the prices of an ordinary digital option and a digital exchange option, respectively. For example, when $y_{0} \rightarrow-\infty$, from the expressions for $G\left(y_{0}\right)$ and $H\left(y_{0}\right)$, the conditional moneyness approximation $G\left(y_{0}\right)+H\left(y_{0}\right) y$ for $A(y)$ becomes

$$
A(y) \approx G(-\infty)+H(-\infty) y=\frac{1}{\nu_{1} \sqrt{1-\rho^{2}}}\left(\mu_{1}-\log K\right)+\frac{\rho}{\sqrt{1-\rho^{2}}} y .
$$

Thus,

$$
e^{-r T} N\left(\frac{G(-\infty)}{\sqrt{1+H^{2}(-\infty)}}\right)=e^{-r T} N\left(\frac{\mu_{1}-\log K}{\nu_{1}}\right)
$$

which is exactly the price of a plain-vanilla digital option with final payoff $1_{S_{1}(T) \geq K}$. Similarly, one can show that the upper bound in equation (26) when $y_{0} \rightarrow+\infty$ is the price of the digital exchange option with final payoff $1_{S_{1}(T) \geq S_{2}(T)}$. Thus the infimum in Proposition 4 automatically incorporates the following inequalities

$$
1_{S_{1}(T) \geq S_{2}(T)+K} \leq 1_{S_{1}(T) \geq K}, \quad \text { and } \quad 1_{S_{1}(T) \geq S_{2}(T)+K} \leq 1_{S_{1}(T) \geq S_{2}(T)} .
$$


This upper bound in Proposition 4 is very tight and can be used as an approximation for the digital spread option price. Numerical analysis shows that the relative pricing errors, defined as the pricing errors divided by the actual prices, are typically of order $10^{-3}$.

In order to make Proposition 4 more useful in practice, we need to have a quick estimate of the optimal value for $y_{0}$ that achieves the infimum in equation (26). The optimal value for $y_{0}$ is given by the solution of the following equation

$$
\frac{\mathrm{d}}{\mathrm{d} y_{0}} N\left(\frac{G\left(y_{0}\right)}{\sqrt{1+H^{2}\left(y_{0}\right)}}\right)=0 .
$$

An analytical solution to the best $y_{0}$ from the above nonlinear equation turns out to be not possible. However, we can linearize equation (30) around $y_{0}=0$ and then solve for the best $y_{0}$ approximately. This should be a very accurate approximation because the best expansion point for $y_{0}$ ought to be very close to 0 since $n(y)$ peaks at 0 . The result is the following choice of $y_{0}$ :

$$
\widehat{y}_{0}=\frac{\xi\left(\lambda \xi+K \nu_{2}\right) \log \left(e^{\mu_{1}} / \xi\right)}{\xi^{2}\left(\nu_{2}^{2}+2 \lambda \nu_{2}-\nu_{1}^{2}\right)-K^{2} \nu_{2}^{2}-2 K \xi \lambda \nu_{2}+e^{\mu_{2}} \xi \lambda \nu_{2} \log \left(e^{\mu_{1}} / \xi\right)},
$$

where

$$
\lambda=\rho \nu_{1}-\nu_{2}, \quad \xi=e^{\mu_{2}}+K
$$

Although the expression of $\widehat{y}_{0}$ is complicated, it is a simple function of the input parameters and can be computed very quickly. Numerical analysis shows that for all reasonable parameter values, we have

$$
0 \leq N\left(\frac{G\left(\widehat{y}_{0}\right)}{\sqrt{1+H^{2}\left(\widehat{y}_{0}\right)}}\right)-\inf _{y_{0} \in \mathbb{R}} N\left(\frac{G\left(y_{0}\right)}{\sqrt{1+H^{2}\left(y_{0}\right)}}\right) \sim 10^{-4} \cdot \inf _{y_{0} \in \mathbb{R}} N\left(\frac{G\left(y_{0}\right)}{\sqrt{1+H^{2}\left(y_{0}\right)}}\right) .
$$

Thus, in practice, we can replace the infimum in Proposition 4 by this particular choice of $\widehat{y}_{0}$ without losing much accuracy.

\section{B. The Lower Bound - Chord Approximation}

A lower bound can also be established for the digital spread option by using the chords of the exercise boundary $\underline{x}(y)$. Pick two points $y_{l}$ and $y_{r}$ on the real line with $y_{r}>y_{l}$. The line passing through points $\left(y_{l}, A\left(y_{l}\right)\right)$ and $\left(y_{r}, A\left(y_{r}\right)\right)$ is given by $B(y)=P\left(y_{l}, y_{r}\right)+Q\left(y_{l}, y_{r}\right) y$, where

$$
P\left(y_{l}, y_{r}\right)=\frac{y_{r} A\left(y_{l}\right)-y_{l} A\left(y_{r}\right)}{y_{r}-y_{l}}, \quad Q\left(y_{l}, y_{r}\right)=\frac{A\left(y_{r}\right)-A\left(y_{l}\right)}{y_{r}-y_{l}} .
$$

The segment of $B(y)$ between points $\left(y_{l}, A\left(y_{l}\right)\right)$ and $\left(y_{r}, A\left(y_{r}\right)\right)$ is a chord of the conditional moneyness function $A(y)$. By Proposition 3, the line $B(y)$ lies below the exercise boundary $\underline{x}(y)$ 
in the region $\left(y_{l}, y_{r}\right)$ and above the exercise boundary $\underline{x}(y)$ outside this region. Because of this, if we directly use $B(y)$ to approximate the conditional moneyness $A(y)$, the resulting price could be either an upper bound or a lower bound. However, notice that we always have

$$
N(A(y)) \geq N(B(y))-[N(A(y))-N(B(y))]^{+} .
$$

This fact can be used to derive a lower bound for the digital spread option given in the following proposition. In the second line of equation (36), we add back two terms by looking at the behavior of the conditional moneyness $A(y)$ in more detail with the help of Proposition 3. The proof of Proposition 5 is in the Appendix.

Proposition 5. Let $K \geq 0$ and $|\rho|<1$. Then

$$
\begin{aligned}
\Pi^{\mathrm{D}} \geq \sup _{\substack{y_{l}, y_{r} \in \mathbb{R} \\
y_{r}>y_{l}}} e^{-r T}\left(N\left(\frac{P\left(y_{l}, y_{r}\right)}{\sqrt{1+Q^{2}\left(y_{l}, y_{r}\right)}}\right)-N\left(y_{l}\right)-N\left(-y_{r}\right)\right. \\
\left.+N\left(-y_{r}\right) N\left(A\left(y_{r}\right)\right) 1_{\rho \geq \nu_{2} / \nu_{1}}+N\left(y_{l}\right) N\left(A\left(y_{l}\right)\right) 1_{\rho \leq 0}\right) .
\end{aligned}
$$

In the above proposition, each candidate in the supremum is the price of a digital option whose exercise boundary is less favorable than the one in our digital spread option. Since the digital spread option price is greater than each of the candidate, it is greater than the supremum of them too. For given values of $y_{l}$ and $y_{r}$, the lower bound can be computed quickly to give an approximation for $\Pi^{D}$.

This lower bound is not as accurate as the upper bound, yielding relative pricing errors of about $2 \%$ in many cases in our numerical analysis. We can also approximate the optimal $y_{l}$ and $y_{r}$ as we have done for the upper bound. We choose not to do it and instead introduce a new approximation which is extremely accurate with relative pricing errors being less than $10^{-4}$ most of the time.

The upper and lower bounds for digital spread options are useful because the delta's and kappa (defined as the option price sensitivity with respect to the spread $K$ ) of a spread option can themselves be considered digital spread option prices, as we will see in Proposition 8 later. Thus our bounds for digital spread options give us bounds on the greeks of spread options.

\section{CLOSED-FORM APPROXIMATIONS FOR SPREAD OP- TION PRICES AND GREEKS}

\section{A. Approximation for Spread Option Prices}

We are now ready to obtain the main result of this paper, that is, a fast and accurate approximation for spread options prices. Our improved approximation is based on a quadratic approximation 
of the exercise boundary and hence a quadratic approximation of the conditional moneyness function $A(y)$. Suppose we approximate $A(y)$ using a parabola by $C^{3}\left(y_{0}\right)+D^{3}\left(y_{0}\right) y+\epsilon\left(y_{0}\right) y^{2}$ around $y=y_{0}$, then

$$
\mathbf{I}_{3}=\int_{-\infty}^{\infty} N(A(y)) n(y) \mathrm{d} y \approx \int_{-\infty}^{\infty} N\left(C^{3}+D^{3} y+\epsilon y^{2}\right) n(y) \mathrm{d} y
$$

The expressions for $C^{3}, D^{3}$ and $\epsilon$ will be given in Proposition 6 . The superscripts 3 in $C^{3}$ and $D^{3}$ indicate that these quantities are for the third term $\mathbf{I}_{3}$. They should not be misinterpreted as powers. We use superscripts instead of subscripts because later we will use subscripts for partial derivatives. The quantities $C^{3}, D^{3}$ and $\epsilon$ are the intercept, slope, and curvature at $y=0$, respectively, of the quadratic approximating boundary $C^{3}\left(y_{0}\right)+D^{3}\left(y_{0}\right) y+\epsilon\left(y_{0}\right) y^{2}$.

By Proposition 2, the last integral in the above equation cannot be evaluated in closed form unless we let $\epsilon=0$. However, if the curvature $\epsilon$ is small around the expansion point $y_{0}$, then we can expand the above integral around $\epsilon=0$. Numerical analysis shows this is indeed the case. Similar observations are made to the integrals $\mathbf{I}_{1}$ and $\mathbf{I}_{2}$. The resulting approximation is given in the following proposition. Proposition 6 approximates $\mathbf{I}_{1}, \mathbf{I}_{2}$ and $\mathbf{I}_{3}$ with a second-order Taylor expansion in terms of the curvature $\epsilon$ of the conditional moneyness function $A(y)$. The proof of Proposition 6 is given in the Appendix.

Proposition 6. Let $K \geq 0$ and $|\rho|<1$. Let $y_{0}$ be any real number close to 0 . The spread option price $\Pi$ under the general jointly-normal returns setup is given by

$$
\Pi=e^{\nu_{1}^{2} / 2+\mu_{1}-r T} \mathbf{I}_{1}-e^{\nu_{2}^{2} / 2+\mu_{2}-r T} \mathbf{I}_{2}-K e^{-r T} \mathbf{I}_{3} .
$$

The integrals $\mathbf{I}_{i}$ 's are approximated to second order in $\epsilon$ as

$$
\mathbf{I}_{i} \approx \mathbf{J}_{0}\left(C^{i}, D^{i}\right)+\mathbf{J}_{1}\left(C^{i}, D^{i}\right) \epsilon+\frac{1}{2} \mathbf{J}_{2}\left(C^{i}, D^{i}\right) \epsilon^{2}
$$

where the function $\mathbf{J}_{i}$ 's are defined as

$$
\begin{aligned}
& \mathbf{J}_{0}(u, v)=N\left(\frac{u}{\sqrt{1+v^{2}}}\right) \\
& \mathbf{J}_{1}(u, v)=\frac{1+\left(1+u^{2}\right) v^{2}}{\left(1+v^{2}\right)^{5 / 2}} \cdot n\left(\frac{u}{\sqrt{1+v^{2}}}\right) \\
& \mathbf{J}_{2}(u, v)=\frac{\left(6-6 u^{2}\right) v^{2}+\left(21-2 u^{2}-u^{4}\right) v^{4}+4\left(3+u^{2}\right) v^{6}-3}{\left(1+v^{2}\right)^{11 / 2}} u \cdot n\left(\frac{u}{\sqrt{1+v^{2}}}\right),
\end{aligned}
$$


and the arguments $C^{i}, D^{i}$, and $\epsilon$ are given by

$$
\begin{aligned}
C^{1} & =C^{3}+D^{3} \rho \nu_{1}+\epsilon \rho^{2} \nu_{1}^{2}+\sqrt{1-\rho^{2}} \nu_{1}, \\
D^{1} & =D^{3}+2 \rho \nu_{1} \epsilon \\
C^{2} & =C^{3}+D^{3} \nu_{2}+\epsilon \nu_{2}^{2} \\
D^{2} & =D^{3}+2 \nu_{2} \epsilon \\
C^{3} & =\frac{1}{\nu_{1} \sqrt{1-\rho^{2}}}\left(\mu_{1}-\log (R+K)+\frac{\nu_{2} R}{R+K} y_{0}-\frac{1}{2} \frac{\nu_{2}^{2} R K}{(R+K)^{2}} y_{0}^{2}\right), \\
D^{3} & =\frac{1}{\nu_{1} \sqrt{1-\rho^{2}}}\left(\rho \nu_{1}-\frac{\nu_{2} R}{R+K}+\frac{\nu_{2}^{2} R K}{(R+K)^{2}} y_{0}\right), \\
\epsilon & =-\frac{1}{2 \nu_{1} \sqrt{1-\rho^{2}}} \frac{\nu_{2}^{2} R K}{(R+K)^{2}},
\end{aligned}
$$

with $R=e^{\nu_{2} y_{0}+\mu_{2}}$.

Note that Proposition 6 also allows us to approximate the digital spread option price $\Pi^{D}$. Systematic numerical analysis demonstrates that a first order approximation in $\epsilon$ by setting $\mathbf{J}_{2}=0$ already yields very accurate spread option prices, although its accuracy seems to be consistently dominated by a second order approximation in $\epsilon$. Moreover, the choice of $y_{0}=0$ works very well and generally produces relative price errors smaller than $10^{-4}$. A zero $y_{0}$ also results in simpler expressions for $C^{i}$ s and $D^{i}$ 's and makes our approximation faster. Thus, we shall fix $y_{0}=0$ throughout this paper. Setting $y_{0}=0$ amounts to matching the slope and curvature of the exercise boundary exactly when the log price of asset two equals its mean.

Our approximation in Proposition 6 has some nice properties. First, it satisfies many boundary conditions. For example, it collapses to the Black-Scholes formula when either $\mu_{1}$ or $\mu_{2}$ approaches $-\infty$. Also, as $\mu_{1}$ goes to infinity, we have $\lim _{\mu_{1} \rightarrow \infty} \Pi / e^{\nu_{1}^{2} / 2+\mu_{1}-r T}=1$, and as $\mu_{2} \rightarrow \infty$, we have $\lim _{\mu_{2} \rightarrow \infty} \Pi=0$. The approximation also satisfies the terminal boundary condition when $T \rightarrow 0$. Second, our approximation collapses exactly to the Margrabe formula when $K=0$ and converges to 0 when $K \rightarrow \infty$. Third, our approximation collapses exactly to the Black-Scholes formula when $\nu_{2} \rightarrow 0$. These nice properties add to the attractiveness of our approximation.

In Section V, we will perform a thorough comparison of our method with other methods in terms of computational speed and accuracy. We shall see that our method is extremely fast and accurate. Before we do that, we study the price sensitivity of spread options. We first perform an analysis for the general jointly-normal return setup in Proposition 7 . We then study the special geometric Brownian motions case and the results are given in Proposition 8 and 9. Readers who are less interested to see the analysis on price sensitivity and greeks can proceed directly to Section V. 


\section{B. Price Sensitivity Analysis of Spread Options}

The price $\Pi$ of the spread option is a function of $\nu_{1}, \nu_{2}, \rho, r, T, \mu_{1}, \mu_{2}$ and $\log K$. Sometimes we view $\Pi$ as a function of $e^{\mu_{1}}, e^{\mu_{2}}$ or $K$ instead of $\mu_{1}, \mu_{2}$, and $K$. For example, in the geometric Brownian motions case, it is usually more natural to look at price sensitivity with respect to $S_{i}$ 's instead of $\log S_{i}$ 's. It should be clear from the context which point of view we use. The sensitivities of spread option prices with respect to these parameters are not well understood under the jointly-normal returns setup, mainly because of the lack of a closed-form formula. We fill this gap with the following two propositions. Proofs are provided in the Appendix.

Proposition 7. Let $K \geq 0$. Under the jointly-normal returns setup, we have:

1. Holding all other variables constant, $\Pi$ is increasing and convex in $e^{\mu_{1}}$.

2. Holding all other variables constant, $\Pi$ is decreasing and convex in $e^{\mu_{2}}$.

3. Holding all other variables constant, $\Pi$ is decreasing and convex in $K$.

4. Holding all other variables constant, $\Pi$ is convex in $\mu_{1}$.

5. Holding all other variables constant, $\Pi$ is decreasing in $\rho$.

6. Holding all other variables constant, $\partial^{2} \Pi / \partial \mu_{1} \partial \mu_{2}<0$.

This proposition generalizes the known price sensitivities of the Black-Scholes formula and the Margrabe formula to the case of spread options. It is easy to understand that $\Pi$ is increasing in $\mu_{1}$, and decreasing in $\mu_{2}$ and $K$. For exchange options, from the Margrabe formula, it is well-known that the option price is convex in $S_{1}$ and $S_{2}$. Proposition 7 generalizes this result to spread options by pointing out that $\Pi$ is convex in $e^{\mu_{1}}, e^{\mu_{2}}$ and $K$. Statement 4 is a new and interesting result. The fact that $\Pi$ decreases with $\rho$ agrees with intuition and generalizes the result for exchange options. Intuitively, for larger values of $\rho$, when the value of asset one increases, the value of asset two tends to increase more, resulting in a smaller value for the spread option.

Notice that under the general jointly-normal returns setup, we cannot say too much on the greeks because we have not specified the functional forms for $\mu_{i}$ 's and $\nu_{i}$ 's. For example, $\mu_{i}$ 's could be complicated functions of the volatilities, time to maturity, etc. Thus, we do not examine the sensitivities of $\Pi$ with respect to $\nu_{i}$ 's in Proposition 7 . However, if we assume geometric Brownian motions for the $S_{i}(t)$ 's, the functional forms of $\mu_{i}$ 's and $\nu_{i}$ 's are determined and we can obtain more results. For simplicity, we assume that $q_{1}=q_{2}=0$ throughout this paper. This does not incur any loss of generality since nonzero $q_{1}$ and $q_{2}$ can be absorbed into a redefinition of $S_{1}$ and $S_{2}$. Notice first that in this special case, equation (5) holds and we have

$$
\Pi=S_{1} \mathbf{I}_{1}-S_{2} \mathbf{I}_{2}-K e^{-r T} \mathbf{I}_{3} .
$$


The following proposition expresses the greeks in terms of one-dimensional integrations and points out the signs of the greeks. These one-dimensional integrals are potentially very useful if one computes the greeks using Monte Carlo simulation. Proposition 8 also characterizes the signs of various greeks for the spread option under the geometric Brownian motions assumption.

Proposition 8. Let $K \geq 0$ and assume that $S_{i}(t)$ 's follow geometric Brownian motions with correlation $\rho$.

1. The sensitivities of the spread option price to initial stock prices $S_{i}$ and spread $K$ are given by

$$
\begin{aligned}
\Delta_{1} & \equiv \frac{\partial \Pi}{\partial S_{1}}=\mathbf{I}_{1}=\int_{-\infty}^{\infty} N\left(A\left(y+\rho \nu_{1}\right)+\sqrt{1-\rho^{2}} \nu_{1}\right) n(y) \mathrm{d} y \\
\Delta_{2} & \equiv \frac{\partial \Pi}{\partial S_{2}}=-\mathbf{I}_{2}=-\int_{-\infty}^{\infty} N\left(A\left(y+\nu_{2}\right)\right) n(y) \mathrm{d} y \\
\kappa & \equiv \frac{\partial \Pi}{\partial K}=-e^{-r T} \mathbf{I}_{3}=-e^{-r T} \int_{-\infty}^{\infty} N(A(y)) n(y) \mathrm{d} y
\end{aligned}
$$

Furthermore, we have

$$
0<\Delta_{1}<1, \quad-1<\Delta_{2}<0, \quad \text { and } \quad-e^{-r T}<\kappa<0
$$

2. The signs of the gamma's are given by

$$
\Gamma_{11} \equiv \frac{\partial^{2} \Pi}{\partial S_{1}^{2}}>0, \quad \Gamma_{12} \equiv \frac{\partial^{2} \Pi}{\partial S_{1} \partial S_{2}}<0, \quad \Gamma_{22} \equiv \frac{\partial^{2} \Pi}{\partial S_{2}^{2}}>0 .
$$

3. The spread option price is a decreasing function of $\rho$ and we have

$$
\frac{\partial \Pi}{\partial \rho}=S_{1} S_{2} \sigma_{1} \sigma_{2} T \Gamma_{12}<0
$$

4. The vega's of the spread option price are given by

$$
\begin{aligned}
\mathcal{V}_{1} \equiv \frac{\partial \Pi}{\partial \sigma_{1}}= & e^{-r T} \sqrt{1-\rho^{2}} \sqrt{T} \int_{-\infty}^{\infty} n(A(y))\left(K+e^{\mu_{2}+\nu_{2} y}\right) n(y) \mathrm{d} y \\
& +\rho S_{1} \sqrt{T} \int_{-\infty}^{\infty} y N\left(A\left(y+\rho \nu_{1}\right)+\sqrt{1-\rho^{2}} \nu_{1}\right) n(y) \mathrm{d} y \\
\mathcal{V}_{2} \equiv \frac{\partial \Pi}{\partial \sigma_{2}}= & -S_{2} \sqrt{T} \int_{-\infty}^{\infty} y N\left(A\left(y+\nu_{2}\right)\right) n(y) \mathrm{d} y .
\end{aligned}
$$

Furthermore, if $\rho \leq 0$, we have $\mathcal{V}_{1} \geq 0$ and $\mathcal{V}_{2} \geq 0$. If $\sigma_{2} / \sigma_{1} \leq \rho \leq 1$, we have $\mathcal{V}_{1} \geq 0$ and $\mathcal{V}_{2} \leq 0$

While the signs of the greeks in the first three statements are well-known, statement 4 gives us some insights on the vegas. It is interesting to note that unlike the Black-Scholes formula, in 
which both call and put option prices are increasing functions of the volatility, the spread option price can be either increasing or decreasing in the two volatilities. For exchange options, from the Margrabe formula, it can be easily shown the sign of $\mathcal{V}_{1}$ is the same as that of $\nu_{1}-\nu_{2} \rho$, and the sign of $\mathcal{V}_{2}$ is the same as that of $\nu_{2}-\nu_{1} \rho$. Proposition 8 is consistent with these results and partially generalizes them to spread options. It is only a partial generalization because the signs of the vegas are still not known when $0 \leq \rho \leq \sigma_{2} / \sigma_{1}$. The difficulty lies in Proposition 3 , which points out that for values of $\rho$ in this range, the conditional moneyness $A(y)$ is no longer monotone in $y$. Besides expressing the greeks in simple one-dimensional integrals, Proposition 8 is useful for other purposes. In particular, bounds on the vega's can be established with the help of Proposition 8. For example, by equation (58), it is easy to show that when $K \geq 0$, we have $\left|\mathcal{V}_{2}\right| \leq S_{2} \sqrt{T} / \sqrt{2 \pi}$.

Finally, in Propositions 7 and 8, we have assumed that $K \geq 0$. For the case $K \leq 0$, we need to use equation (12) to reduce to the $K \geq 0$ case. The modifications are as follows. For Proposition 7, all statements are still true when $K \leq 0$ except statement 4 . Most expressions in Proposition 8 can no longer be directly used when $K \leq 0$. The correct expressions in this case can be worked out easily with the help of equation (12). Because this process is not completely trivial, we use $\kappa$ as an illustration. Writing $\Pi=\Pi\left(S_{1}, S_{2}, \sigma_{1}, \sigma_{2}, \rho, K\right)$, we have by equation (12) that

$$
\Pi\left(S_{1}, S_{2}, \sigma_{1}, \sigma_{2}, \rho, K\right)=S_{1}-S_{2}-K e^{-r T}+\Pi\left(S_{2}, S_{1}, \sigma_{2}, \sigma_{1}, \rho,-K\right) .
$$

We will call the above equation the generalized put-call parity for spread options. Indeed, if $S_{2}=0$ and $\sigma_{2}=0$, then the above relation reduces to the put-call parity for plain-vanilla European options in the Black-Scholes formula. If $K=0$, then the above equation reduces to the put-call parity for exchange options in the Margrabe formula. Now let $K \leq 0$ in equation (59). To compute $\kappa$, we have by the generalized put-call parity and Proposition 8 that

$$
\begin{aligned}
\kappa & =\frac{\partial}{\partial K} \Pi\left(S_{1}, S_{2}, \sigma_{1}, \sigma_{2}, \rho, K\right)=-e^{-r T}+\frac{\partial}{\partial K} \Pi\left(S_{2}, S_{1}, \sigma_{2}, \sigma_{1}, \rho,-K\right) \\
& =-e^{-r T}+e^{-r T} \int_{-\infty}^{\infty} N\left(A^{\dagger}(x)\right) n(x) \mathrm{d} x=-e^{-r T} \int_{-\infty}^{\infty} N\left(-A^{\dagger}(x)\right) n(x) \mathrm{d} x,
\end{aligned}
$$

where $A^{\dagger}(x)$ is the mirror image of $A(y)$ given as

$$
A^{\dagger}(x)=\frac{\rho x-\underline{y}(x)}{\sqrt{1-\rho^{2}}}
$$

with

$$
\underline{y}(x)=\frac{\log \left(e^{\nu_{1} x+\mu_{1}}+|K|\right)-\mu_{2}}{\nu_{2}} .
$$


Equations (53) and (60) seem to give two different expressions for $\kappa$ when $K=0$. They actually lead to exactly the same result if we evaluate them using Proposition 2. The reason that the function $A^{\dagger}(x)$ now gets involved is that by using the put-call parity, the roles of asset one and asset two get switched. Other greeks can be modified similarly when $K \leq 0$ and are omitted here. It is worthwhile pointing out that when $K \leq 0$, the expression for $\mathcal{V}_{1}$ now involves only one integral while the expression for $\mathcal{V}_{2}$ now involves two integrals. Also, equations (54), (55) and (56) in Proposition 8 are still correct. The last sentence in statement 4 when $K \leq 0$ should be changed to: If $\sigma_{1} / \sigma_{2} \leq \rho \leq 1$, we have $\mathcal{V}_{1} \leq 0$ and $\mathcal{V}_{2} \geq 0$. Again, it is easy to check that this new statement is consistent with the known behavior of $\mathcal{V}_{1}$ and $\mathcal{V}_{2}$ for the exchange option case.

\section{Approximations for the Greeks in the GBMs Case}

Proposition 6 gives the approximate price for the spread option in closed form. However, in practice one often needs to compute the greeks efficiently. This can be done by differentiating the approximate price $\Pi$ in Proposition 6. However, the algebra is quite complicated. Instead, we design separate approximations for the greeks in the special geometric Brownian motions case in Proposition 9 below. This proposition allows for fast and accurate computation of the greeks for the spread option in this important special case. The proof relies on Proposition 8 and is again based heavily on the boundary approximation. It is given in the Appendix.

Proposition 9. Let $K \geq 0$ and assume that $S_{i}(t)$ 's follow geometric Brownian motions with correlation $\rho$.

1. The delta's and kappa can be approximated as

$$
\begin{aligned}
\Delta_{1} & \approx \mathbf{J}_{0}\left(C^{1}, D^{1}\right)+\mathbf{J}_{1}\left(C^{1}, D^{1}\right) \epsilon+\frac{1}{2} \mathbf{J}_{2}\left(C^{1}, D^{1}\right) \epsilon^{2}, \\
\Delta_{2} & \approx-\mathbf{J}_{0}\left(C^{2}, D^{2}\right)-\mathbf{J}_{1}\left(C^{2}, D^{2}\right) \epsilon-\frac{1}{2} \mathbf{J}_{2}\left(C^{2}, D^{2}\right) \epsilon^{2}, \\
\kappa & \approx-\mathbf{J}_{0}\left(C^{3}, D^{3}\right)-\mathbf{J}_{1}\left(C^{3}, D^{3}\right) \epsilon-\frac{1}{2} \mathbf{J}_{2}\left(C^{3}, D^{3}\right) \epsilon^{2} .
\end{aligned}
$$

2. The gamma's can be approximated as

$$
\begin{aligned}
& \Gamma_{11} \approx \Phi\left(C^{1}, D^{1}, C_{S_{1}}^{1}, D_{S_{1}}^{1}, \epsilon_{S_{1}}\right), \\
& \Gamma_{12} \approx \Phi\left(C^{1}, D^{1}, C_{S_{2}}^{1}, D_{S_{2}}^{1}, \epsilon_{S_{2}}\right), \\
& \Gamma_{22} \approx-\Phi\left(C^{2}, D^{2}, C_{S_{2}}^{2}, D_{S_{2}}^{2}, \epsilon_{S_{2}}\right),
\end{aligned}
$$

where the function $\Phi$ is defined by

$$
\Phi(x, y, u, v, w)=n\left(\frac{x}{\sqrt{1+y^{2}}}\right) \frac{\left(1+y^{2}\right)^{2} u-x\left(y+y^{3}\right) v+\left(1+\left(1+x^{2}\right) y^{2}\right) w}{\left(1+y^{2}\right)^{5 / 2}},
$$


and the partial derivatives are given by

$$
C_{S_{1}}^{1}=\frac{1}{\nu_{1} S_{1} \sqrt{1-\rho^{2}}}, \quad D_{S_{1}}^{1}=0, \quad \epsilon_{S_{1}}=0
$$

and

$$
\begin{aligned}
C_{S_{2}}^{1} & =\frac{\epsilon}{S_{2}}\left(\frac{2(R+K)}{\nu_{2}^{2} K}+\frac{2 \rho \nu_{1}}{\nu_{2}}+\left(1-\frac{2 R}{R+K}\right) \rho^{2} \nu_{1}^{2}\right), \\
D_{S_{2}}^{1} & =\frac{\epsilon}{S_{2}}\left(\frac{2}{\nu_{2}}+2 \rho \nu_{1}\left(1-\frac{2 R}{R+K}\right)\right), \\
C_{S_{2}}^{2} & =\frac{\epsilon}{S_{2}}\left(\frac{2(R+K)}{\nu_{2}^{2} K}+2+\left(1-\frac{2 R}{R+K}\right) \nu_{2}^{2}\right), \\
D_{S_{2}}^{2} & =\frac{\epsilon}{S_{2}}\left(\frac{2}{\nu_{2}}+2 \nu_{2}\left(1-\frac{2 R}{R+K}\right)\right), \\
\epsilon_{S_{2}} & =\frac{\epsilon}{S_{2}}\left(1-\frac{2 R}{R+K}\right) .
\end{aligned}
$$

3. The approximation for vega with respect to $\sigma_{1}$ is given by

$$
\begin{aligned}
\mathcal{V}_{1} \approx S_{1} \Phi & \left(C^{1}, D^{1}, C_{\sigma_{1}}^{1}, D_{\sigma_{1}}^{1}, \epsilon_{\sigma_{1}}\right) \\
& -S_{2} \Phi\left(C^{2}, D^{2}, C_{\sigma_{1}}^{2}, D_{\sigma_{1}}^{2}, \epsilon_{\sigma_{1}}\right)-K e^{-r T} \Phi\left(C^{3}, D^{3}, C_{\sigma_{1}}^{3}, D_{\sigma_{1}}^{3}, \epsilon_{\sigma_{1}}\right),
\end{aligned}
$$

where

$$
\begin{array}{ll}
C_{\sigma_{1}}^{1}=-\frac{C^{3}}{\sigma_{1}}+\epsilon \rho^{2} \sigma_{1} T, & D_{\sigma_{1}}^{1}=D_{\sigma_{1}}^{3}, \\
C_{\sigma_{1}}^{2}=C_{\sigma_{1}}^{3}+D_{\sigma_{1}}^{3} \nu_{2}+\epsilon_{\sigma_{1}} \nu_{2}^{2}, & D_{\sigma_{1}}^{2}=D_{\sigma_{1}}^{3}+2 \nu_{2} \epsilon_{\sigma_{1}}, \\
C_{\sigma_{1}}^{3}=-\frac{\sqrt{T}}{\sqrt{1-\rho^{2}}}-\frac{C^{3}}{\sigma_{1}}, & D_{\sigma_{1}}^{3}=-\frac{\sigma_{2}}{\sigma_{1}^{2} \sqrt{1-\rho^{2}}} \frac{R}{R+K}, \\
\epsilon_{\sigma_{1}}=-\frac{\epsilon}{\sigma_{1}} . &
\end{array}
$$

The approximation for vega with respect to $\sigma_{2}$ is given by

$$
\mathcal{V}_{2} \approx S_{2} \sqrt{T} \Upsilon\left(C^{2}, D^{2}, \epsilon\right),
$$

where the function $\Upsilon$ is given by

$$
\Upsilon(u, v, w)=n\left(\frac{u}{\sqrt{1+v^{2}}}\right) v \frac{\left(1+v^{2}\right)^{3}+u w\left(3+\left(3+u^{2}\right) v^{2}\right)}{\left(1+v^{2}\right)^{7 / 2}} .
$$

Although we do not discuss them here, higher-order greeks such as the vomma's and vanna's (defined as the sensitivities of option vegas with respect to volatilities and spot prices, respectively) can be easily approximated as well using our method. Our approximations for the greeks involve only simple arithmetic and are very fast to compute. Also, they are derived using the same idea as for the price approximation and are thus extremely accurate, often giving relative errors well within $0.1 \%$. 


\section{COMPARISON OF ACCURACY AND SPREAD WITH EX- ISTING METHODS}

\section{A. Existing Approximation Methods}

Various methods have been proposed for pricing spread options, which can be broadly divided into two groups. The first group follows the numerical approach and includes Pearson (1995)'s one-dimensional numerical integration method, fast Fourier transform methods as in Dempster and Hong (2000), numerical solutions to partial differential equations, and Monte Carlo methods. The second group tries to approximate the spread option price and greeks analytically, and includes Bachelier approximation, Bachelier approximation with Gram-Charlier adjustment, Kirk's approximation, Carmona-Durrleman approximation, and others. Eydeland and Wolyniec (2003) also contain a closed-form approximation for spread options, which is not very accurate. In this section, we will compare the speed and accuracy of our method with alternative methods. We first discuss some of the alternative methods, namely, the Bachelier approximation, Bachelier approximation with Gram-Charlier adjustment, Kirk's approximation and the Carmona-Durrleman approximation. In the next subsection, we then perform a comparison of speed and accuracy of existing methods with our approximation.

In the Bachelier approximation, the quantity $B \equiv e^{-r T}\left(S_{1}(T)-S_{2}(T)\right)$ is approximated as a normal random variable, with mean $\mu_{B}$ and standard deviation $\sigma_{B}$ given by

$$
\mu_{B}=S_{1}-S_{2}, \quad \sigma_{B}=\sqrt{S_{1}^{2}\left(\theta_{1}-1\right)-2 S_{1} S_{2}\left(\theta_{2}-1\right)+S_{2}^{2}\left(\theta_{3}-1\right)}
$$

where

$$
\theta_{1}=e^{\sigma_{1}^{2} T}, \quad \theta_{2}=e^{\sigma_{2}^{2} T}, \quad \theta_{3}=e^{\rho \sigma_{1} \sigma_{2} T}
$$

The spread option price is then approximated using the Bachelier formula for a plain-vanilla European call option (Bachelier 1900) as

$$
\Pi_{\mathrm{B}}=\left(\mu_{B}-K e^{-r T}\right) N\left(d_{B}\right)+\sigma_{B} n\left(d_{B}\right)
$$

where

$$
d_{B}=\frac{\mu_{B}-K e^{-r T}}{\sigma_{B}} .
$$

The assumption that $B$ is normal is very crude. Mbafeno (1997) proposes a skewness and kurtosis adjustment based on the Gram-Charlier approximation in Jarrow and Rudd (1982). However, the formula in Mbafeno (1997) contains a typo. Also, he does not give explicit expressions for the skewness and kurtosis under the GBMs setup. Thus, we present the result for the 
Gram-Charlier approximation in the following proposition, which takes into account the nonzero skewness $\gamma_{B}$ and excess kurtosis $\kappa_{B}$ explicitly. The proof is given in the Appendix.

Proposition 10. Suppose that the asset prices $S_{1}(t)$ and $S_{2}(t)$ follow geometric Brownian motions with initial prices $S_{1}$ and $S_{2}$. Assume that $B \equiv e^{-r T}\left(S_{1}(T)-S_{2}(T)\right)$ follows a Gram-Charlier approximation which includes skewness and kurtosis adjustments. Then the approximate spread option prices is

$$
\Pi_{\mathrm{GC}}=\left(\mu_{B}-K e^{-r T}\right) N\left(d_{B}\right)+\sigma_{B} n\left(d_{B}\right)\left(1-\frac{d_{B}}{6} \gamma_{B}+\frac{d_{B}^{2}-1}{24} \kappa_{B}\right),
$$

with skewness $\gamma_{B}$ and excess kurtosis $\kappa_{B}$ given by

$$
\gamma_{B}=\frac{\mathbb{E}^{\mathbb{Q}}\left(B-\mu_{B}\right)^{3}}{\sigma_{B}^{3}}, \quad \kappa_{B}=\frac{\mathbb{E}^{\mathbb{Q}}\left(B-\mu_{B}\right)^{4}}{\sigma_{B}^{4}}-3
$$

where

$$
\begin{aligned}
\mathbb{E}^{\mathbb{Q}}\left(B-\mu_{B}\right)^{3}= & \left(\theta_{1}-1\right)^{2}\left(\theta_{1}+2\right) S_{1}^{3}-\left(\theta_{2}-1\right)^{2}\left(\theta_{2}+2\right) S_{2}^{3} \\
& -3\left(\theta_{3}-1\right)\left(\theta_{1}+\theta_{1} \theta_{3}-2\right) S_{1}^{2} S_{2}+3\left(\theta_{3}-1\right)\left(\theta_{2}+\theta_{2} \theta_{3}-2\right) S_{1} S_{2}^{2},
\end{aligned}
$$

and

$$
\begin{aligned}
& \mathbb{E}^{\mathbb{Q}}\left(B-\mu_{B}\right)^{4}=\left(6 \theta_{1}-4 \theta_{1}^{3}+\theta_{1}^{6}-3\right) S_{1}^{4}+\left(6 \theta_{2}-4 \theta_{2}^{3}+\theta_{2}^{6}-3\right) S_{2}^{4} \\
& \quad+4\left(3-3 \theta_{1}+\theta_{1}^{3}-3 \theta_{3}-\theta_{1}^{3} \theta_{3}^{3}+3 \theta_{1} \theta_{3}^{2}\right) S_{1}^{3} S_{2}+4\left(3-3 \theta_{2}+\theta_{2}^{3}-3 \theta_{3}-\theta_{2}^{3} \theta_{3}^{3}+3 \theta_{2} \theta_{3}^{2}\right) S_{1} S_{2}^{3} \\
& \quad+6\left(\theta_{1}+\theta_{2}+3 \theta_{3}-2 \theta_{2} \theta_{3}^{2}-2 \theta_{1} \theta_{3}^{2}+\theta_{1} \theta_{2} \theta_{3}^{4}-3\right) S_{1}^{2} S_{2}^{2} .
\end{aligned}
$$

Except for the fact that the expressions for the skewness $\gamma_{B}$ and excess kurtosis $\kappa_{B}$ are complicated, equation (87) in Proposition 10 is a standard result. For example, it is well-known that the skewness adjustment is proportional to the moneyness $d_{B}$ while the excess kurtosis adjustment is quadratic in $d_{B}$. Unlike the Bachelier approximation, in the Gram-Charlier approximation the spread option price can become negative, especially when the skewness and excess kurtosis are large. Whenever this happens, we will assume the approximated spread option price is 0. Also, as many researchers have noticed, it is possible for the Gram-Charlier approximation to perform worse than the Bachelier approximation if the deviation of $B$ from normality is significant. We found that this is indeed the case.

In Kirk's approximation, $Z(T)=S_{2}(T)+K$ is considered as a lognormal random variable. The initial value of $Z$ is given by $z=S_{2}+K e^{-r T}$. The volatility for $Z(T)$ is value weighted using the relative proportions of the stock and bond positions and is given by

$$
\sigma_{Z}=\frac{S_{2}}{S_{2}+K e^{-r T}} \sigma_{2}
$$


The correlation of $S_{1}(T)$ and $Z(T)$ are still $\rho$. Kirk then uses the Margrabe formula to price the spread option (now viewed as a simple exchange option):

$$
\begin{aligned}
\Pi_{\mathrm{K}} & =e^{-r T} \mathbb{E}^{\mathbb{Q}}\left[S_{1}(T)-S_{2}(T)-K\right]^{+} \approx e^{-r T} \mathbb{E}^{\mathbb{Q}}\left[S_{1}(T)-Z(T)\right]^{+} \\
& =S_{1} N\left(d_{K}+\sigma_{K} \sqrt{T} / 2\right)-\left(S_{2}+K e^{-r T}\right) N\left(d_{K}-\sigma_{K} \sqrt{T} / 2\right),
\end{aligned}
$$

where

$$
d_{K}=\frac{\log \left(S_{1} /\left(S_{2}+K e^{-r T}\right)\right.}{\sigma_{K} \sqrt{T}}, \quad \sigma_{K}=\sqrt{\sigma_{1}^{2}-2 \rho \sigma_{1} \sigma_{2} \frac{S_{2}}{S_{2}+K e^{-r T}}+\sigma_{2}^{2}\left(\frac{S_{2}}{S_{2}+K e^{-r T}}\right)^{2}} .
$$

Although not obvious, Kirk's approximation can be thought of as a rough version of our approximation, in which the exercise boundary is approximated using three straight lines very close to it. Details are available upon request from the authors. Kirk's approximation is relatively accurate given its simple form, with relative price errors usually within a few percentages.

More recently, a new approximation formula is proposed in Carmona and Durrleman (2003a, $2003 \mathrm{~b})$. In this method, one first solves the following equation to get an optimal $\theta^{*}$ :

$$
\begin{aligned}
\frac{1}{\sigma_{2} \sqrt{T} \cos \theta} & \log \left(-\frac{\sigma_{1} K e^{-r T} \sin (\theta+\phi)}{S_{2}\left(\sigma_{1} \sin (\theta+\phi)-\sigma_{2} \sin \theta\right)}\right)+\frac{\sigma_{2} \sqrt{T} \cos \theta}{2} \\
& =\frac{1}{\sigma_{1} \sqrt{T} \cos (\theta+\phi)} \log \left(-\frac{\sigma_{2} K e^{-r T} \sin \theta}{S_{1}\left(\sigma_{1} \sin (\theta+\phi)-\sigma_{2} \sin \theta\right)}\right)+\frac{\sigma_{1} \sqrt{T} \cos (\theta+\phi)}{2}
\end{aligned}
$$

where $\phi=\arccos (\rho)$. Now let

$$
d^{*}=\frac{1}{\sigma \sqrt{T} \cos \left(\theta^{*}-\psi\right)} \log \left(\frac{S_{1} \sigma_{1} \sin \left(\theta^{*}+\phi\right)}{S_{2} \sigma_{2} \sin \theta^{*}}\right)+\frac{1}{2}\left(\sigma_{1} \cos \left(\theta^{*}+\phi\right)+\sigma_{2} \cos \theta^{*}\right) \sqrt{T},
$$

where

$$
\sigma=\sqrt{\sigma_{1}^{2}-2 \rho \sigma_{1} \sigma_{2}+\sigma_{2}^{2}}, \quad \psi=\arccos \left(\frac{\sigma_{2}-\rho \sigma_{1}}{\sigma}\right)
$$

In the above equations, we have corrected a few typos in Carmona and Durrleman (2003a, 2003b), ranging from missing minus signs and switching of cos and sin functions. Carmona and Durrleman (2003a, 2003b) show that the spread option price is always larger than the following lower bound

$$
\Pi_{\mathrm{CD}}=S_{1} N\left(d^{*}+\sigma_{1} \sqrt{T} \cos \left(\theta^{*}+\phi\right)\right)-S_{2} N\left(d^{*}+\sigma_{2} \sqrt{T} \cos \theta^{*}\right)-K e^{-r T} N\left(d^{*}\right) .
$$

In addition, they show that the lower bound is very tight and can be used as a good approximation for the actual price. Our numerical analysis confirms their claim. It is also noteworthy to point out that recently Carmona and Durrleman (2006) have extended their results to multiple assets cases. 
Our method improves upon existing approximations in many aspects. First, previous approximations have focused on the spread option as a single identity and developed formulas for the whole spread option, while in our method, we approximate the three individual terms separately. Each individual term in our method is approximated extremely accurately. This contrasts many previous methods. For example, the accuracy of Kirk's method relies on a delicate cancelation of errors between the three terms in equation (93). The individual terms in Kirk's approximation are very inaccurate, often having relative pricing errors reaching 200\%. Approximating individual terms also allows us to compute digital-type spread options, such as asset-or-nothing and cash-or-nothing spread options. Although we do not develop formulas in this paper, our boundary approximation method also allows us to compute more exotic spread options, for example, options with quadratic payoff $S_{1}(T)^{2} 1_{S_{1}(T) \geq S_{2}(T)+K}$.

Second, and perhaps the most significant advantage of our method, is that we provide extremely accurate and fast approximations for the greeks. For example, delta's in our method often have relative errors in the order of $0.1 \%$.

Third, our method achieves a good balance between speed and accuracy. It is more accurate than the Bachelier approximation and Kirk's method. While the Carmona-Durrleman approximation is more accurate than Kirk's approximation, it is still about one or two orders' of magnitude less accurate than our method. Furthermore, like most analytical methods, our method is very fast. The computing times of Bachelier approximation, Kirk's approximation and ours are roughly of the same order of magnitude. However, the Carmona-Durrleman approximation is slower than most analytical approximations since one needs to solve a complicated trigonometrical equation to get the optimal $\theta^{*}$. This is time-consuming even if one utilizes fast algorithms such as the Newton-Raphson method.

Finally, our approximation is very straightforward to implement and is very robust. We find that it is not trivial to find $\theta^{*}$ for equation (95) in the Carmona-Durrleman approximation because sometimes the objective function is not smooth in $\theta^{*}$ and there is little guidance on the initial value for $\theta$. Thus, it is hard to use the Newton-Raphson method. The lack of guidance on the initial value for $\theta$ also prevents us from comparing systematically the performance of CarmonaDurrleman approximation with ours. The important case $K=0$ also poses some problems in Carmona-Durrleman because equation (95) breaks down. Another issue is that whenever $\theta^{*}$ is the solution to equation (95), so is $\theta^{*}+\pi$. Only one of the two solutions will correspond to the lower bound. Lacking any guidance on how to select $\theta^{*}$ and $\theta^{*}+\pi$, one would have to compute option prices for both solutions and then compare. Thus, the Carmona-Durrleman approximation is not as straightforward as our method. 


\section{B. Comparison of Accuracy and Speed with Existing Methods}

For definiteness, we shall assume the special GBMs case and compare our method with Bachelier approximation, Bachelier approximation with Gram-Charlier adjustment, Kirk's approximation and one-dimensional numerical integration method. The one-dimensional numerical integration is based on Proposition 1.

Notice that the spread option price is a function of many variables, namely, $S_{1}, S_{2}, T, r, q_{1}$, $q_{2}, \sigma_{1}, \sigma_{2}, K$, and $\rho$. We will set $q_{1}=q_{2}=0$ as nonzero $q_{i}$ 's can be absorbed into $S_{1}$ and $S_{2}$. Also, since $\sigma_{i}$ 's and $\sqrt{T}$ always go together, we set $T=1$. Because the spread option price is homogeneous of degree 1 in $S_{1}, S_{2}$ and $K$, we fix $S_{1}=100$. The interest rate $r$ usually does not play a major role so we fix it at $5 \%$. In order to perform a systematic accuracy comparison, we vary $S_{2} / S_{1}, K / S_{1}, \sigma_{1}, \sigma_{2}$ and $\rho$. We set the range of $S_{2} / S_{1}$ to be $[0.7,1.2]$, the range of $K / S_{1}$ to be $[0,0.4]$, the range of $\sigma_{1}$ and $\sigma_{2}$ to be both [0.1,0.8], and the range of $\rho$ to be $[-0.75,0.75]$. In addition, we impose the restriction $S_{1}-S_{2}-K e^{-r T} \geq-30$ so that we exclude deeply out-of-themoney options. Parameters are uniformly generated from these ranges. All together, we generate 123,783 individual options. Generating options uniformly will tend to weigh extreme scenarios more heavily and thus tend to exaggerate an approximation method's pricing errors.

We then compute the spread option prices for those 123,783 options using Bachelier approximation, Bachelier approximation with Gram-Charlier adjustment, Kirk's approximation and the approximation in this paper. We also compute the prices using one-dimensional numerical integration with recursive adaptive Simpson quadrature method. Two error tolerance levels are used: $10^{-6}$ and $10^{-8}$. The prices computed from the latter tolerance level are then used as the actual option prices. All methods are implemented in MATLAB 7.0 on an IBM computer with 1.60 GHz Intel Pentium CPU and 768M memory and some other computers. The computing times of different methods do not seem to vary with the specification of the computer on which they are run. For each option and for each approximation method, we first compute the the pricing error $\Delta \Pi=\Pi_{\text {Approximation }}-\Pi_{\text {Actual }}$ and then compute the relative pricing error $\Delta \Pi / \Pi_{\text {Actual }}$. Because the actual prices vary greatly from 0 to around 65 , we focus on the relative pricing errors.

\section{Insert Exhibit 2 Approximately Here}

The results are reported in Exhibit 2. For the numerical methods, we do not list their accuracy since it has been specified. Looking at $\Delta \Pi / \Pi_{\text {Actual }}$, Bachelier approximation gives quite inaccurate spread option prices. Also, Bachelier approximation with Gram-Charlier adjustment seems 
to perform worse than no adjustment. Detailed analysis shows that the deviations of $B$ from normality are often quite significant, with mean excess kurtosis for those 123,783 options being around 10. In addition, Bachelier approximation and Kirk's approximation seem to be biased upward. Most of the time, they give a price larger than the actual price. The bias in our method seems to be extremely small. Also, we see that our method is extremely accurate, with a median $\left|\Delta \Pi / \Pi_{\text {Actual }}\right|$ of $3.8 \times 10^{-6}$. In terms of speed, Bachelier approximation and Kirk's approximation are the fastest while our method is not too far behind. In particular, we see that our method is capable of computing one million spread option prices within 10 seconds. The numerical integration methods are much slower, with computing times roughly two orders of magnitude larger than those of the analytical methods.

\section{CONCLUSION}

In this paper, we first obtain lower and upper bounds for digital spread options by analyzing the exercise boundary. We then develop a new closed-form approximation for pricing spread options. Numerical analysis demonstrates that our method is more accurate than existing analytical approximations. It is also extremely fast, capable of computing one million spread options within 10 seconds. Thus, our method enables the accurate pricing of a bulk volume of spread options with different specifications in real time which offers traders a potential edge in financial markets. The availability of a closed-form formula for spread options also helps us design and analyze real and financial contracts with embedded spread-option-like features.

We also derive closed-form approximations for the greeks of spread options. The closed-form approximations of greeks serve as valuable tools in financial applications. For instance, they can be used for calculating Value-at-Risk for a portfolio containing spread options. As byproducts, we analyze the price sensitivities of spread options. In particular, we point out the signs of vegas when the correlation is negative and when the correlation is positive and large. The analysis of the price sensitivities leads to improved understanding of the price behavior of spread options and is useful in formulating effective dynamic-hedging strategies.

There are a few directions that one can take to extend and improve the results in this paper. First, Deng, Li and Zhou (2008) show that one can extend the boundary approximation method for two-asset spread options to the multiple assets case. The extended approximation is shown to be extremely fast and accurate. Second, our approximation is useful even if one wants to incorporate jumps in the price processes of the assets. Carmona and Durrleman (2003b) discuss in detail how the approximated price can be used in such cases. Third, Li (2008) studies the correction to the exchange option price when the asset returns deviate from the jointly normal 
distribution using a bivariate Gram-Charlier approximation. His method can be extended to the spread option case if we couple it with our exercise boundary approximation. This will allow the pricing of spread options under arbitrary distributions that are close to jointly-normal. Finally, it is possible to further improve the accuracy of our method. For example, in our spread option approximation, we have set the expansion point to be $y_{0}=0$, that is, we have expanded around the point where the future log price of asset two equals its mean. This is a degree of freedom which we have not utilized. Preliminary numerical analysis suggests that the optimal expansion point $y_{0}$ seems to depend on the correlation coefficient $\rho$. Thus, relaxing $y_{0}$ may make our approximation even more accurate, especially when $|\rho|$ is large. We leave these issues to future research. 


\section{APPENDIX}

\section{Proof of Proposition 1:}

The random variables $X$ and $Y$ are jointly normally distributed. Denote this density $n(x, y ; \rho)$. The conditional density of $X$ given $Y=y$ is $n\left(x ; \rho y, 1-\rho^{2}\right)$, i.e., a normal density with mean $\rho y$ and variance $1-\rho^{2}$. Thus, we can now compute the price of the digital spread option as follows:

$$
\begin{aligned}
\Pi^{\mathrm{D}} & =e^{-r T} \int_{-\infty}^{\infty} \int_{-\infty}^{\infty} 1_{x \geq \underline{x}(y)} n(x, y ; \rho) \mathrm{d} x \mathrm{~d} y=e^{-r T} \int_{-\infty}^{\infty} n(y) \mathrm{d} y \int_{\underline{x}}^{\infty} n\left(x ; \rho y, 1-\rho^{2}\right) \mathrm{d} x \\
& =e^{-r T} \int_{-\infty}^{\infty} n(y) N(A(y)) \mathrm{d} y .
\end{aligned}
$$

Similarly, the spread option price can be computed as

$$
\begin{aligned}
\Pi & =e^{-r T} \int_{-\infty}^{\infty} \int_{-\infty}^{\infty}\left(e^{\nu_{1} x+\mu_{1}}-e^{\nu_{2} y+\mu_{2}}-K\right)^{+} n(x, y ; \rho) \mathrm{d} x \mathrm{~d} y \\
& =e^{-r T} \int_{-\infty}^{\infty} n(y) \mathrm{d} y \int_{\underline{x}}^{\infty}\left(e^{\nu_{1} x+\mu_{1}}-e^{\nu_{2} y+\mu_{2}}-K\right) n\left(x ; \rho y, 1-\rho^{2}\right) \mathrm{d} x .
\end{aligned}
$$

By virtue of the identity

$$
\int_{x_{0}}^{\infty} e^{t x} n\left(x ; \mu, \sigma^{2}\right) \mathrm{d} x=e^{\mu t+\sigma^{2} t^{2} / 2} N\left(\frac{\mu-x_{0}}{\sigma}+\sigma t\right),
$$

the inner integral can be performed to yield

$$
\begin{aligned}
\Pi= & e^{-r T} \int_{-\infty}^{\infty} n(y) e^{\rho y \nu_{1}+\left(1-\rho^{2}\right) \nu_{1}^{2} / 2+\mu_{1}} N\left(A(y)+\sqrt{1-\rho^{2}} \nu_{1}\right) \mathrm{d} y \\
& -e^{-r T} \int_{-\infty}^{\infty} n(y) e^{\nu_{2} y+\mu_{2}} N(A(y)) \mathrm{d} y \\
& -e^{-r T} \int_{-\infty}^{\infty} n(y) K N(A(y)) \mathrm{d} y .
\end{aligned}
$$

It is very useful to perform a change of variable to the above equation to obtain

$$
\begin{aligned}
\Pi= & e^{\nu_{1}^{2} / 2+\mu_{1}-r T} \int_{-\infty}^{\infty} n(y) N\left(A\left(y+\rho \nu_{1}\right)+\sqrt{1-\rho^{2}} \nu_{1}\right) \mathrm{d} y \\
& -e^{\nu_{2}^{2} / 2+\mu_{2}-r T} \int_{-\infty}^{\infty} n(y) N\left(A\left(y+\nu_{2}\right)\right) \mathrm{d} y . \\
& -K e^{-r T} \int_{-\infty}^{\infty} n(y) N(A(y)) \mathrm{d} y \\
\equiv & e^{\nu_{1}^{2} / 2+\mu_{1}-r T} \mathbf{I}_{1}-e^{\nu_{2}^{2} / 2+\mu_{2}-r T} \mathbf{I}_{2}-K e^{-r T} \mathbf{I}_{3} .
\end{aligned}
$$

Mathematically, this is similar to a change of numeraire. 


\section{Proof of Proposition 2:}

Define

$$
F(a) \equiv \int_{-\infty}^{\infty} N(a+y) n(y) \mathrm{d} y, \quad G(b) \equiv \int_{-\infty}^{\infty} N(a+b y) n(y) \mathrm{d} y
$$

Notice that

$$
F(0)=\int_{-\infty}^{\infty} N(y) n(y) \mathrm{d} y=\int_{-\infty}^{\infty} N(y) \mathrm{d} N(y)=\frac{1}{2}
$$

and

$$
F^{\prime}(a)=\int_{-\infty}^{\infty} n(a+y) n(y) \mathrm{d} y=\frac{1}{2 \sqrt{\pi}} \exp \left(-\frac{a^{2}}{4}\right)=\frac{1}{\sqrt{2}} n\left(\frac{a}{\sqrt{2}}\right)
$$

Thus,

$$
F(a)=F(0)+\int_{0}^{a} F^{\prime}(a) \mathrm{d} a=N\left(\frac{a}{\sqrt{2}}\right)
$$

We can compute

$$
G(1)=F(a)=N\left(\frac{a}{\sqrt{2}}\right), \quad \text { and } \quad G^{\prime}(b)=-\frac{a b}{\left(1+b^{2}\right)^{3 / 2}} n\left(\frac{a}{\sqrt{1+b^{2}}}\right) .
$$

Thus

$$
\int_{-\infty}^{\infty} N(a+b y) n(y) \mathrm{d} y=G(b)=G(1)+\int_{1}^{b} G^{\prime}(b) \mathrm{d} b=N\left(\frac{a}{\sqrt{1+b^{2}}}\right) .
$$

The first integral in the lemma now follows immediately:

$$
\int_{-\infty}^{\infty} N(a+b y) n\left(y ; \mu, \sigma^{2}\right) \mathrm{d} y=\int_{-\infty}^{\infty} N(a+b \mu+b \sigma z) n(z) \mathrm{d} z=N\left(\frac{a+b \mu}{\sqrt{1+b^{2} \sigma^{2}}}\right) .
$$

To prove the Margrabe formula, notice that when $K=0$, we have

$$
\underline{x}(y)=\frac{\nu_{2} y+\mu_{2}-\mu_{1}}{\nu_{1}}, \quad A(y)=\frac{\mu_{1}-\mu_{2}}{\nu_{1} \sqrt{1-\rho^{2}}}+\frac{\left(\rho \nu_{1}-\nu_{2}\right)}{\nu_{1} \sqrt{1-\rho^{2}}} y \equiv a+b y .
$$

By Proposition 1, we need to compute $\mathbf{I}_{1}$ and $\mathbf{I}_{2}$. Since the conditional moneyness $A(y)$ is linear, we can use equation (19). For example, to compute $\mathbf{I}_{2}$, we have

$$
\begin{aligned}
\mathbf{I}_{2} & =\int_{-\infty}^{\infty} N\left(A\left(y+\nu_{2}\right)\right) n(y) \mathrm{d} y=\int_{-\infty}^{\infty} N(A(y)) n\left(y ;-\nu_{2}, 1\right) \mathrm{d} y \\
& =\int_{-\infty}^{\infty} N(a+b y) n\left(y ;-\nu_{2}, 1\right) \mathrm{d} y=N\left(\frac{a-b \nu_{2}}{\sqrt{1+b^{2}}}\right)=N\left(\frac{\mu_{1}-\mu_{2}-\left(\nu_{2}^{2}-\rho \nu_{1} \nu_{2}\right)}{\sqrt{\nu_{1}^{2}+\nu_{2}^{2}-2 \rho \nu_{1} \nu_{2}}}\right) .
\end{aligned}
$$

Similarly for $\mathbf{I}_{1}$. 


\section{Proof of Proposition 3:}

This proposition follows from pure algebraic manipulation. In particular, we need to examine the first and second-order derivatives of $\underline{x}(y), A(y)$ and $N(A(y))$ with respect to $y$.

\section{Proof of Proposition 4:}

This proposition follows immediately from equation (26) and Proposition 1.

\section{Proof of Proposition 5:}

With $B(y)$ defined in the text, we have

$$
\begin{aligned}
\mathbf{I}_{3} & =\int_{-\infty}^{\infty} n(y) N(A(y)) \mathrm{d} y=\int_{-\infty}^{\infty} n(y) N(B(y)) \mathrm{d} y+\int_{-\infty}^{\infty} n(y)(N(A(y))-N(B(y))) \mathrm{d} y \\
& \geq \int_{-\infty}^{\infty} n(y) N(B(y)) \mathrm{d} y-\int_{-\infty}^{\infty} n(y)(N(A(y))-N(B(y)))^{+} \mathrm{d} y \\
& =N\left(\frac{P}{\sqrt{1+Q^{2}}}\right)-\int_{-\infty}^{y_{l}} n(y)(N(A(y))-N(B(y))) \mathrm{d} y-\int_{y_{r}}^{\infty} n(y)(N(A(y))-N(B(y))) \mathrm{d} y .
\end{aligned}
$$

The upper bound is approximated by replacing $N(B(y))$ with 1 . When $\rho \leq 0$ or $\rho \geq \nu_{2} / \nu_{1}$, we can use Proposition 3 to tighten up the bound.

\section{Proof of Proposition 6:}

We will consider the last term for the spread option first.

$$
\mathbf{I}_{3}=\int_{-\infty}^{\infty} n(y) N(A(y)) \mathrm{d} y \approx \int_{-\infty}^{\infty} n(y) N\left(C^{3}+D^{3} y+\epsilon y^{2}\right) \mathrm{d} y .
$$

This integral can not be performed. However, if the curvature $\epsilon$ is small around the expansion point $y=y_{0}$, then we can expand the above integral around $\epsilon=0$. Since,

$$
\begin{aligned}
& \frac{\mathrm{d} N\left(C^{3}+D^{3} y+\epsilon y^{2}\right)}{\mathrm{d} \epsilon}=n\left(C^{3}+D^{3} y+\epsilon y^{2}\right) y^{2}, \\
& \frac{\mathrm{d}^{2} N\left(C^{3}+D^{3} y+\epsilon y^{2}\right)}{\mathrm{d} \epsilon^{2}}=-\left(C^{3}+D^{3} y+\epsilon y^{2}\right) n\left(C^{3}+D^{3} y+\epsilon y^{2}\right) y^{4},
\end{aligned}
$$

we have

$$
\mathbf{I}_{3} \approx \mathbf{J}_{0}+\mathbf{J}_{1} \epsilon+\frac{1}{2} \mathbf{J}_{2} \epsilon^{2}
$$


where

$$
\begin{aligned}
& \mathbf{J}_{0}=\int_{-\infty}^{\infty} n(y) N\left(C^{3}+D^{3} y\right) \mathrm{d} y, \\
& \mathbf{J}_{1}=\int_{-\infty}^{\infty} n(y) n\left(C^{3}+D^{3} y\right) y^{2} \mathrm{~d} y, \\
& \mathbf{J}_{2}=-\int_{-\infty}^{\infty} n(y)\left(C^{3}+D^{3} y\right) n\left(C^{3}+D^{3} y\right) y^{4} \mathrm{~d} y,
\end{aligned}
$$

The $\mathbf{J}_{i}$ 's can be computed to give the expressions in the Proposition. In particular, $\mathbf{J}_{0}$ can be computed using Proposition 2. The integrals $\mathbf{I}_{1}$ and $\mathbf{I}_{2}$ can be treated similarly. However, in Proposition 1, the expansion points for $\mathbf{I}_{1}$ and $\mathbf{I}_{2}$ are chosen to be $y_{0}-\rho \nu_{1}$ and $y_{0}-\nu_{2}$, respectively. This amounts to using the same expansion point $y_{0}$ for all three terms in equation (102).

\section{Proof of Proposition 7:}

The first four statements can be proven by differentiating either equations (104) to (106) or the expression for $\Pi$ in Proposition 1. The algebra is tedious and omitted here. Statement 5 and 6 are shown in Carmona and Durrleman (2003a).

\section{Proof of Proposition 8:}

Notice that in the geometric Brownian motions cases, we have equation (5) for the $\mu_{i}$ 's. The first statement follows from directly differentiating the expression of $\Pi$ in Proposition 1 . Notice that by definition, when $x=\underline{x}(y)$, we have $e^{\sigma_{1} \sqrt{T} x+\mu_{1}}-e^{\sigma_{2} \sqrt{T} y+\mu_{2}}-K=0$. Statement 2 follows from Proposition 7 directly. A proof of statement 3 is contained in Carmona and Durrleman (2003a). Finally, statement 4 follows from directly differentiating the expression of $\Pi$ in Proposition 1 and simplifying. We will take $\mathcal{V}_{2}$ as an example. Notice that for the geometric Brownian motions case, equation (102) becomes

$$
\Pi=e^{-r T} \int_{-\infty}^{\infty} n(y) \mathrm{d} y \int_{\underline{x}}^{\infty}\left(e^{\nu_{1} x+\mu_{1}}-e^{\sigma_{2} \sqrt{T} y+\left(r-q-\sigma_{2}^{2} / 2\right) T}-K\right) n\left(x ; \rho y, 1-\rho^{2}\right) \mathrm{d} x .
$$

The derivative of $\Pi$ with respect to $\sigma_{2}$ has two terms: one arising from the dependence of the lower inner integration limit $\underline{x}(y)$ on $\sigma_{2}$ and another arising from the term $e^{\sigma_{2} \sqrt{T} y+\left(r-q-\sigma_{2}^{2} / 2\right) T}$. Differentiating on the integration limit gives 0 since by definition of the exercise boundary

$$
e^{\nu_{1} \underline{x}(y)+\mu_{1}}-e^{\sigma_{2} \sqrt{T} y+\left(r-q-\sigma_{2}^{2} / 2\right) T}-K=0 .
$$

Thus,

$$
\begin{aligned}
\frac{\partial \Pi}{\partial \sigma_{2}} & =-e^{-r T} \int_{-\infty}^{\infty} n(y) \sqrt{T}\left(y-\sigma_{2} \sqrt{T}\right) \mathrm{d} y \int_{\underline{x}}^{\infty} e^{\sigma_{2} \sqrt{T} y+\left(r-q-\sigma_{2}^{2} / 2\right) T} n\left(x ; \rho y, 1-\rho^{2}\right) \mathrm{d} x \\
& =-e^{-r T} \int_{-\infty}^{\infty} n(y) \sqrt{T}\left(y-\sigma_{2} \sqrt{T}\right) e^{\sigma_{2} \sqrt{T} y+\left(r-q-\sigma_{2}^{2} / 2\right) T} N(A(y)) \mathrm{d} y .
\end{aligned}
$$


Completing the square like we did in the proof of Proposition 1 now gives

$$
\frac{\partial \Pi}{\partial \sigma_{2}}=-S_{2} \sqrt{T} \int_{-\infty}^{\infty} n\left(y ; \sigma_{2} \sqrt{T}, 1\right)\left(y-\sigma_{2} \sqrt{T}\right) N(A(y)) \mathrm{d} y .
$$

Finally, a change of variable gives the final expression for $\mathcal{V}_{2}$ in the proposition

$$
\frac{\partial \Pi}{\partial \sigma_{2}}=-S_{2} \sqrt{T} \int_{-\infty}^{\infty} n(y) y N\left(A\left(y+\sigma_{2} \sqrt{T}\right)\right) \mathrm{d} y .
$$

The expression for $\mathcal{V}_{1}$ is more complicated because differentiating $\Pi$ in equation (131) with respect to $\sigma_{1}$ will give

$$
\frac{\partial \Pi}{\partial \sigma_{1}}=e^{-r T} \int_{-\infty}^{\infty} n(y) \mathrm{d} y \int_{\underline{x}}^{\infty} \sqrt{T}\left(x-\nu_{1}\right) e^{\nu_{1} x+\mu_{1}} n\left(x ; \rho y, 1-\rho^{2}\right) \mathrm{d} x
$$

and the factor $x-\nu_{1}$ contributes to the inner integration. If we now let $z \equiv(x-\rho y) / \sqrt{1-\rho^{2}}$, then we have

$$
\frac{\partial \Pi}{\partial \sigma_{1}}=\sqrt{T} e^{-r T} \int_{-\infty}^{\infty} n(y) e^{\rho \nu_{1} y+\mu_{1}} \mathrm{~d} y \int_{-A(y)}^{\infty}\left(\sqrt{1-\rho^{2}} z+\rho y-\nu_{1}\right) e^{\sqrt{1-\rho^{2}} \nu_{1} z} n(z) \mathrm{d} z .
$$

By virtue of equation (103),

$$
\int_{-A(y)}^{\infty} e^{\sqrt{1-\rho^{2}} \nu_{1} z} n(z) \mathrm{d} z=e^{\nu_{1}^{2}\left(1-\rho^{2}\right) / 2} N\left(A(y)+\nu_{1} \sqrt{1-\rho^{2}}\right) .
$$

Integration by parts using $n^{\prime}(z)=-z n(z)$ gives

$$
\int_{-A(y)}^{\infty} z e^{\sqrt{1-\rho^{2}} \nu_{1} z} n(z) \mathrm{d} z=e^{\nu_{1}(\underline{x}(y)-\rho y)} n(A(y))+\nu_{1} \sqrt{1-\rho^{2}} e^{\nu_{1}^{2}\left(1-\rho^{2}\right) / 2} N\left(A(y)+\nu_{1} \sqrt{1-\rho^{2}}\right) .
$$

Substituting the last two equations into (138), simplifying, and performing a last change of variable $w=y-\rho \nu_{1}$ gives the expression for $\mathcal{V}_{1}$ in the proposition.

For the signs of vegas, notice that $\mathcal{V}_{1}$ is positive whenever

$$
\begin{aligned}
& \rho \int_{-\infty}^{\infty} n(y) y N\left(A\left(y+\rho \nu_{1}\right)+\sqrt{1-\rho^{2}} \nu_{1}\right) \mathrm{d} y \\
& \quad=\rho \int_{0}^{\infty} n(y) y\left(N\left(A\left(y+\rho \nu_{1}\right)+\sqrt{1-\rho^{2}} \nu_{1}\right)-N\left(A\left(-y+\rho \nu_{1}\right)+\sqrt{1-\rho^{2}} \nu_{1}\right)\right) \mathrm{d} y
\end{aligned}
$$

is positive. By Proposition 3, $\rho N\left(A\left(y+\rho \nu_{1}\right)+\sqrt{1-\rho^{2}} \nu_{1}\right)$ is an increasing function when $\rho \leq 0$ or when $\rho \geq \sigma_{2} / \sigma_{1}$. From the above equation, $\mathcal{V}_{1} \geq 0$ when $\rho \leq 0$ or when $\rho \geq \sigma_{2} / \sigma_{1}$. Similarly,

$$
\begin{aligned}
\mathcal{V}_{2} \equiv \frac{\partial \Pi}{\partial \sigma_{2}}= & -S_{2} \sqrt{T} \int_{-\infty}^{\infty} n(y) y N\left(A\left(y+\nu_{2}\right)\right) \mathrm{d} y \\
& =-S_{2} \sqrt{T} \int_{0}^{\infty} n(y) y\left(N\left(A\left(y+\nu_{2}\right)\right)-N\left(A\left(-y+\nu_{2}\right)\right)\right) \mathrm{d} y
\end{aligned}
$$


This integral is positive when $\rho \geq 0$ and negative when $\rho \geq \sigma_{2} / \sigma_{1}$. Alternatively, one could apply Chebyshev's algebraic inequality on equation (142). See, for example, Chapter IX of Mitrinovic, Pecaric and Fink (1992).

\section{Proof of Proposition 9:}

Statement 1 follows from Proposition 8. For statement 2, we will only derive the approximation for $\Gamma_{11}$ since the other gamma's are similar. We have

$$
\begin{aligned}
\Gamma_{11} & \equiv \frac{\partial^{2} \Pi}{\partial S_{1}^{2}}=\frac{\partial \Delta_{1}}{\partial S_{1}}=\frac{\partial}{\partial S_{1}} \int_{-\infty}^{\infty} n(y) N\left(A\left(y+\rho \nu_{1}\right)+\sqrt{1-\rho^{2}} \nu_{1}\right) \mathrm{d} y \\
& \approx \frac{\partial}{\partial S_{1}} \int_{-\infty}^{\infty} n(y) N\left(C^{1}+D^{1} y+\epsilon y^{2}\right) \mathrm{d} y \\
& \approx \int_{-\infty}^{\infty} n(y) n\left(C^{1}+D^{1} y+E^{\prime} y^{2}\right)\left(C_{S_{1}}^{1}+D_{S_{1}}^{1} y+\epsilon_{S_{1}} y^{2}\right) \mathrm{d} y \\
& \approx \int_{-\infty}^{\infty} n(y) n\left(C^{1}+D^{1} y\right)\left(C_{S_{1}}^{1}+D_{S_{1}}^{1} y+\epsilon_{S_{1}} y^{2}\right) \mathrm{d} y=\Phi\left(C^{1}, D^{1}, C_{S_{1}}^{1}, D_{S_{1}}^{1}, \epsilon_{S_{1}}\right),
\end{aligned}
$$

where the function $\Phi$ can be computed analytically to give the expression in the proposition. The greek $\mathcal{V}_{1}$ can be computed similarly. The approximation for $\mathcal{V}_{2}$ is developed similarly using the last statement in Proposition 8.

\section{Proof of Proposition 10:}

This proposition follows from brute-force calculation. We also verified the statements using both Mathematica and MATLAB. We sketch the proof below. Let $Z=\left(B-\mu_{B}\right) / \sigma_{B}$ be the standardized random variable of $B$. Then the Gram-Charlier density for $Z$ is given by

$$
f_{Z}^{\mathrm{GC}}(z)=n(z)\left(1+\frac{\gamma_{B}}{3 !} h_{3}(z)+\frac{\kappa_{B}}{4 !} h_{4}(z)\right)
$$

where $h_{3}(\cdot)$ and $h_{4}(\cdot)$ are Hermite polynomials of order 3 and 4 , respectively. The option price under this Gram-Charlier density can be computed by direct integration to give equation (87). Finally, the following fact is useful in the computation of $\gamma_{B}$ and $\kappa_{B}$. Let $X$ and $Y$ be jointly normal with means $\mu_{X}$ and $\mu_{Y}$, variances $\sigma_{X}^{2}$ and $\sigma_{Y}^{2}$, and correlation coefficient $\rho$. For any real numbers $t$ and $s$, the joint moment generating function is given by

$$
\mathbb{E}\left[e^{t X+s Y}\right]=\exp \left(t \mu_{X}+s \mu_{Y}+\frac{1}{2} t^{2} \sigma_{X}^{2}+\frac{1}{2} s^{2} \sigma_{Y}^{2}+\rho s t \sigma_{X} \sigma_{Y}\right) .
$$




\section{Endnotes}

The authors thank Stephen Figlewski (the editor) for valuable comments and suggestions. We also thank seminar participants at the 2007 Financial Management Association Annual Meeting and the Georgia Tech Quantitative and Computational Finance seminar for useful discussions. Feedbacks from academic scholars, especially Neil Pearson at UIUC and Rene Carmona at Princeton, and practitioners, especially Krzysztof Wolyniec at Sampra Commodities, Sundeep Jain at UBS, and Gary Kennedy at Calypso are greatly appreciated. Kyuseok Lee provided valuable assistance during this project. All remaining errors are ours. 


\section{References}

Albizzati, M-O., and H. Geman. "Interest rate risk management and valuation of the surrender option in life insurance policies." Journal of Risk and Insurance, 61, No. 4 (1994), pp. 616-637.

Arak, M., P. Fisher, L. Goodman, and R. Daryanani. "The municipal-treasury futures spread." Journal of Futures Markets, 7 (1987), pp. 355-371.

Black, F., and M. Scholes. "The pricing of options and corporate liabilities." Journal of Political Economy, 81 (1973), pp. 637-659.

Broadie, M., and J. Detemple. "American option valuation: New bounds, approximations, and a comparison of existing methods." Review of Financial Studies, 9, No. 4 (1996), pp. 12111250.

Carmona, R., and V. Durrleman. "Pricing and hedging spread options." SIAM Review, 45, No. 4 (2003a), pp. 627-685.

—. "Pricing and hedging spread options in a lognormal model." Working paper, 2003b.

- "Generalizing the Black-Scholes formula to multivariate contingent claims." Journal of Computational Finance, 9, No. 2 (2006), pp. 43-67.

Chen, R-R., and S-K. Yeh. "Analytical upper bounds for American option prices." Journal of Financial and Quantitative Analysis, 37, No. 1 (2002), pp. 117-136.

Chung, S-L., and H-C. Chang. "Generalized analytical upper bounds for American option prices." Journal of Financial and Quantitative Analysis, 42, No. 1 (March 2007), pp. 209228.

Dempster, M., and S. Hong. "Pricing spread options with the Fast Foruier Transform." First World Congress of the Bachelier Finance Society, Paris, 2000.

Deng, S.J., B. Johnson, and A. Sogomonian. "Exotic electricity options and the valuation of electricity generation and transmission assets." Decision Support Systems, 30, No. 3 (2001), pp. 383-392.

Deng, S.J., M. Li, and J. Zhou. "Multi-asset spread option pricing and hedging." Working paper, 2008.

Easterwood, J.C., and A.J. Senchack Jr. "Arbitrage opportunities with T-Bills/T-Bonds combinations." Journal of Futures Markets. 6 (1986), pp. 433-442.

Eydeland, A., and K. Wolyniec. Energy and Power Risk Management: New Developments in Modeling, Pricing and hedging. John Wiley \& Sons, New York, 2003.

Geman, H., N. E. Karoui, and J. C. Rochet. "Changes of numeraire, changes of probability measure and option pricing." Journal of Applied Probability, 32 (1995), pp. 443-458.

Girma, P.B., and A.S. Paulson, "Seasonality in petroleum futures spreads." Journal of Futures Markets, 18 (1998), pp. 581-598.

—. "Risk arbitrage opportunities in petroleum futures spreads." Journal of Futures Markets, 18 (1999), pp. 931-955.

Grinblatt, M., and S. Titman. "Adverse risk incentives and the design of performance-based contracts." Management Science, 35, No. 7 (July 1989), pp. 807-822.

Henderson, V., D. Hobson, W.T. Shaw, and R. Wojakowski. "Bounds for in-progress floatingstrike Asian options using symmetry." Annals of Operations Research, 151, No. 1 (April 2007), pp. 81-98. 
Jarrow, R., and A. Rudd. "Approximate option valuation for arbitrary stochastic processes." Journal of Financial Economics, 10 (1982), pp. 347-369.

Johnson, S.A., and Y.S. Tian. "Indexed executive stock options." Journal of Financial Economics, 57 (2000), pp. 35-64.

Johnson, R.L., C.R. Zulauf, S.H. Irwin and M.E. Gerlow. "The soy-bean complex spread: An examination of Market Efficiency from the viewpoint of a production process." Journal of Futures Markets, 11 (1991), pp. 25-37.

Jones, F.J. "Spreads: Tails, turtles and all that." Journal of Futures Markets, 11 (1991), pp. 565-596.

Kirk, E. "Correlations in the energy markets, in managing energy price risk." Risk Publications and Enron, 1995.

Li, M. "The impact of return nonnormality on exchange options." Journal of Futures Markets, forthcoming, 2008.

Lo, A. "Semiparametric upper bounds for option prices and expected payoffs." Journal of Financial Economics 19 (1987), pp. 373-388.

Margrabe, W. "The value of an option to exchange one asset for another." Journal of Finance, 33 (1978), pp. 177-186.

Mbafeno, A. "Co-movement term structure and the valuation of energy spread options." In M. Dempster and S. Pliska, eds., Mathematics of Derivative Securities. Cambridge University Press, Cambridge, UK, 1997.

McDonald, R. L., and D. R. Siegel. "Investment and the valuation of firms when there is an option to shut down." International Economic Review, 26, No. 2 (June 1985), pp. 331-349.

Mitrinovic, D.S., J. Pecaric, and A.M. Fink. Classical and New Inequalities in Analysis. Springer, New York, 1992.

Nielsen, J.A., and K. Sandmann. "Pricing bounds on Asian options." Journal of Financial and Quantitive Analysis, 38, No. 2. (2003), pp. 449-473.

Pearson, N. "An efficient approach for pricing spread options." Journal of Derivatives, (Fall 1995), pp. 76-91.

Perrakis, S., and P.J. Ryan. "Option pricing bounds in discrete time." Journal of Finance, 39 (1984), pp. 519-525.

Poitras, G. "Spread options, exchange options, and arithmetic Brownian motion." Journal of Futures Markets, 18 (1998), pp. 487-517.

Shevlin, T. "The valuation of R\&D firms with R\&D limited parterships." The Accounting Review, 66, No. 1 (Jan. 1991), pp. 1-21.

Shimko, D. "Options on future spreads: Hedging, Speculating, and Valuation." Journal of Futures Markets, 14, No. 2 (1997), pp. 183-213.

Wilcox, D. "Energy futures and options: Spread options in energy markets." Goldman Sachs \& Co., New York, 1990. 
EXHIBIT 1

The Exercise Boundary $\underline{x}(y)$

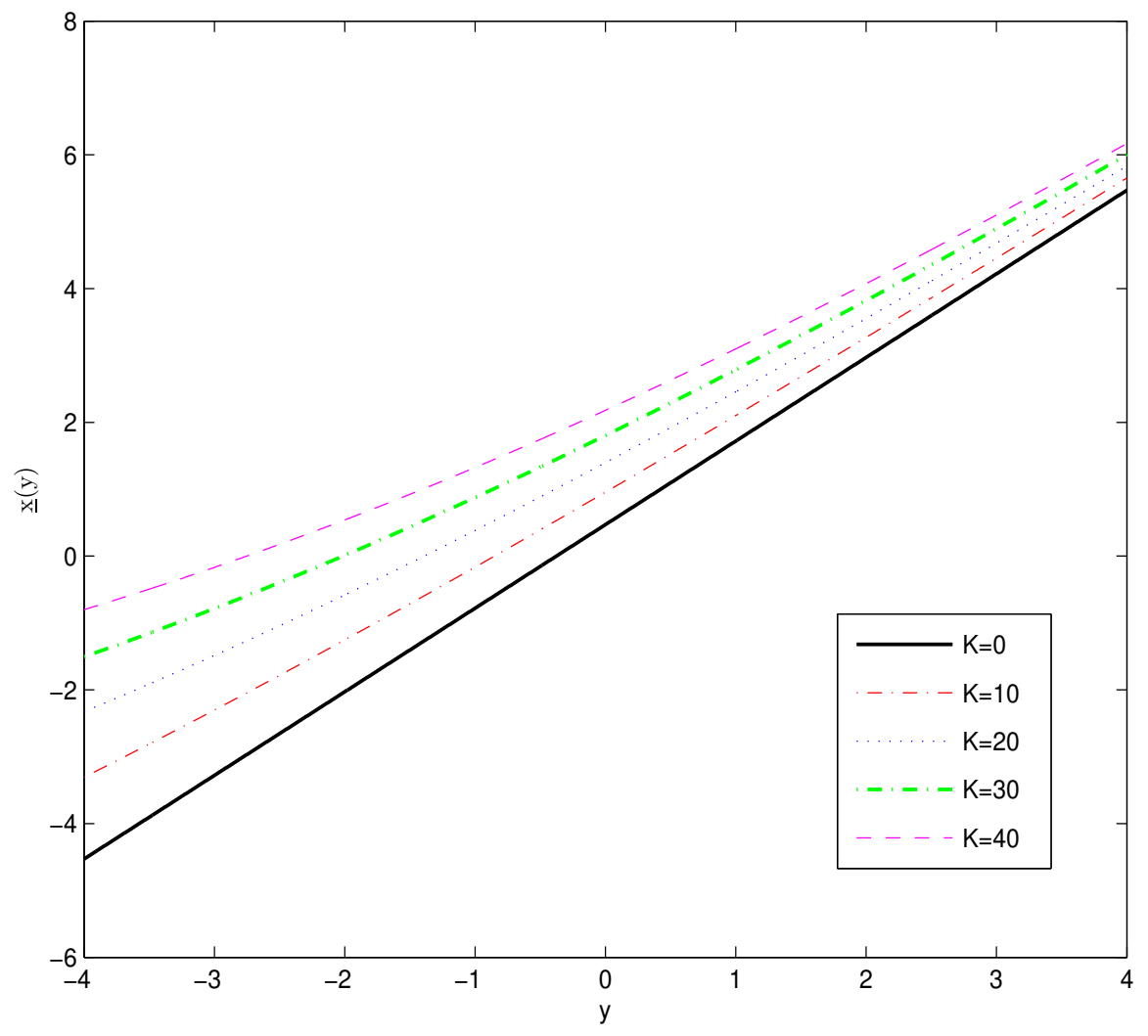




\section{ExhIBIT 2}

\section{Performance Comparison of Various Methods in Computing Spread Option Prices}

This exhibit reports the speed and accuracy of Bachelier approximation, Bachelier approximation with Gram-Charlier adjustment, Kirk's approximation, the approximation in this paper and one-dimensional numerical integration. Total number of options is 123,783. NI stands for numerical integration.

\begin{tabular}{|c|c|c|c|c|c|c|}
\hline & \multicolumn{6}{|c|}{ Methods } \\
\hline & Ours & Bachelier & Gram-Charlier & Kirk & $\mathrm{NI}-10^{-6}$ & $\mathrm{NI}-10^{-8}$ \\
\hline \multicolumn{7}{|l|}{$\Delta \Pi / \Pi_{\text {Actual }}$} \\
\hline $\max$ & 0.027 & 2.558 & 0.411 & 0.460 & & \\
\hline $\min$ & -0.030 & -0.621 & -1.000 & -0.018 & & \\
\hline mean & $-5.1 \times 10^{-5}$ & 0.139 & -0.337 & 0.007 & & \\
\hline median & $-1.3 \times 10^{-7}$ & 0.117 & -0.209 & $9.1 \times 10^{-4}$ & & \\
\hline std. deviation & $7.6 \times 10^{-4}$ & 0.142 & 0.338 & 0.017 & & \\
\hline \multicolumn{7}{|l|}{$\left|\Delta \Pi / \Pi_{\text {Actual }}\right|$} \\
\hline $\max$ & 0.030 & 2.558 & 1.000 & 0.460 & & \\
\hline $\min$ & $\sim 10^{-15}$ & $2.5 \times 10^{-6}$ & $1.0 \times 10^{-7}$ & $\sim 10^{-15}$ & & \\
\hline mean & $1.7 \times 10^{-4}$ & 0.147 & 0.338 & 0.008 & & \\
\hline median & $3.8 \times 10^{-6}$ & 0.119 & 0.209 & 0.0015 & & \\
\hline std. deviation & $7.4 \times 10^{-4}$ & 0.133 & 0.337 & 0.016 & & \\
\hline time (seconds) & 1.02 & 0.22 & 1.12 & 0.25 & 363.57 & 891.60 \\
\hline
\end{tabular}

